\title{
Quirópteros do Parque Estadual da Pedra Branca, Rio de Janeiro, Brasil (Mammalia, Chiroptera)
}

\author{
Daniela Dias ${ }^{1}$ \\ Adriano Lúcio Peracchi ${ }^{1}$ \\ Shirley Seixas Pereira da Silva ${ }^{1}$
}

\begin{abstract}
Bats from Pedra Branca State Park, Rio de Janeiro, Brazil (Mammalia, Chiroptera). A survey of chiropteran fauna was conducted during the period from March 1994 to May 1998 in Pedra Branca State Park, Rio de Janeiro, Rio de Janeiro State. A total of 681 specimens of 24 species were recorded: Chrotopterus auritus (Peters, 1856); Micronycteris megalotis (Gray, 1842); Micronycteris minuta (Gervais, 1856); Mimmon bennettii (Gray, 1838); Phyllostomus hastatus (Pallas, 1767); Tonatia bidens (Von Spix, 1823); Lonchophylla bokermanni Sazima, Vizotto \& Taddei, 1978; Lonchophylla mordax Thomas, 1903; Anoura caudifera (E. Geoffroy, 1818); Glossophaga soricina (Pallas, 1766); Carollia perspicillata (Linnaeus, 1758); Artibeus fimbriatus Gray, 1838; Artibeus lituratus (Olfers, 1818); Artibeus obscurus Schinz, 1821; Chiroderma doriae Thomas, 1891; Platyrrhinus lineatus (E. Geoffroy, 1810); Platyrrhinus recifinus (Thomas, 1901); Sturnira lilium (E. Geoffroy, 1810); Vampyressa pusilla (Wagner, 1843); Desmodus rotundus (E. Geoffroy, 1810); Diphylla ecaudata Von Spix, 1823; Eptesicus brasiliensis (Desmarest, 1819); Myotis nigricans (Schinz, 1821) and Molossus molossus (Palas, 1766). One external (forearm length) and 13 cranial meansurements were studied for 23 species. The meansurements of male and female specimens were treated separately. Comments about some taxonomic respects for some species studied are also included.
\end{abstract}

KEY WORDS. Chiroptera, morphometrics, systematics, Southeastern Brazil

A Ordem Chiroptera distribui-se pela maior parte das regiões temperadas e tropicais, de ambos os hemisférios, estando ausente apenas em ilhas oceânicas remotas e regiões polares (NOWAK 1991). KOOPMAN (1993) reconheceu 925 espécies de morcegos, incluídas em 17 famílias. Todavia, em nenhuma outra região encontra-se a grande diversidade de espécies que caracteriza a região Neotropical, onde 266 espécies de morcegos foram listadas (WILSON 1996). Na América do Sul, onde KoOpMAn (1993) mencionou a ocorrência de, pelo menos, 190 espécies, distribuídas por nove famílias, a fauna de quirópteros ainda é pouco conhecida do ponto de vista taxonômico e zoogeográfico.

Com relação ao Brasil, onde 139 espécies têm registros confirmados (BERNARD 2001), o conhecimento sobre a sistemática de quirópteros ainda é restrito.

Na região Sudeste, poucos são os estudos sistemáticos, abordando profundamente a morfometria das espécies. Nesse aspecto, destacam-se nesta região as importantes contribuições de TADDEI (1975a,b; 1979), sobre Phyllostomidae da região norte-ocidental do Estado de São Paulo.

1) Laboratório de Mastozoologia, Instituto de Biologia, Universidade Federal Rural do Rio de Janeiro. Antiga Rodovia Rio-São Paulo, Km 47, 23890-000 Seropédica, Rio de Janeiro, Brasil. E-mail: ddtdani@ig.com.br 
No Estado do Rio de Janeiro, onde se localiza a área aqui estudada, o conhecimento taxonômico infelizmente é ainda mais defasado, levando-se ainda em conta que o Estado encontra-se dentro da área de abrangência da província da Mata Atlântica, segundo a divisão zoogeográfica proposta por KOOPMAN (1978; 1982) para a América do Sul. SiLVEIRA (1965) relacionou 35 espécies de morcegos para a região Sudeste, mas não forneceu informações precisas quanto às localidades. Estudos sobre quirópteros no Estado do Rio de Janeiro foram subsequentemente conduzidos, abordando dados sobre hábitos alimentares, reprodução e outros aspectos bionômicos, entre os quais os mais importantes são PERACCHI \& ALBUQUERQUE (1971; 1986), ÁVILA-PIRES \& GOUVÊA (1977), VAZ (1985), TEIXEIRA \& PERACCHI (1996) e ESBERÁRD (1998). Estes trabalhos não incluíram dados morfométricos ou outras informações sobre a sistemática dos morcegos.

O Parque Estadual da Pedra Branca constitui um dos últimos trechos conservados de Mata Atlântica no Estado do Rio de Janeiro, onde esse bioma, como em outros estados, vem sofrendo severa devastação. Percebe-se, nesse sentido, a importância do Parque, uma vez que sua cobertura vegetal, no que se refere a abrigos e recursos alimentares, constitui um importante suporte para a manutenção de diversas populações animais (OliveIRA et al. 1995).

Pelo acima exposto e visando contribuir para o conhecimento da quiropterofauna do Estado do Rio de Janeiro, constituem objetivos deste trabalho: 1) proceder ao levantamento das espécies de morcegos que ocorrem no Parque Estadual da Pedra Branca, localizado no município do Rio de Janeiro; 2) fornecer dados morfométricos sobre as espécies inventariadas e; 3) discutir aspectos relacionados à taxonomia de algumas das espécies estudadas.

\section{Área de estudo}

O Parque Estadual da Pedra Branca (PEPB) localiza-se na Zona Oeste do Município do Rio de Janeiro (Rio de Janeiro), entre $23^{\circ} 52^{\prime}$ e $23^{\circ} 04^{\prime} \mathrm{S}$ e $43^{\circ} 23^{\prime}$ e $43^{\circ} 32^{\prime}$ W. Distribuindo-se por 12.500 hectares, no Maciço da Pedra Branca, é a Unidade de Conservação Ambiental mais extensa do município, abrangendo encostas localizadas acima da cota altimétrica de 100 metros (IBAM 1998). O Parque Estadual da Pedra Branca (PEPB), hoje sob tutela da Fundação Instituto Estadual de Florestas (IEF/RJ), foi criado pela Lei Estadual n² 2377, de 28 de junho de 1974 (IBAM 1998).

De acordo com a classificação de Koppen, o clima predominante corresponde ao tipo Af, quente e úmido (OliveIra et al. 1980). A área do Maciço da Pedra Branca, onde se localiza o PEPB, inclui-se na área de abrangência da Província Florestal Atlântica, Setor da Cordilheira Marítima (FERnANDES \& BEZERRA 1990). O Maciço ainda é um trecho bem conservado, sendo possível encontrar espécies raras como os jequitibás (Cariniana estrellensis e C. legalis) e o tapinhoã (Mezilaurus navalium), endêmicas como a noz-moscada-silvestre (Cryptocarya jacarepaguensis), encontrada somente no Município do Rio de Janeiro, e ameaçadas de extinção como o palmiteiro (Euterpe edulis), segundo estudo publicado pelo IBAM (1998), que cita também a existência de espécies introduzidas como o cafeeiro (Coffea arabica), a jabuticabeira (Myrciaria cauliflora), a jaqueira (Artocarpus heterophilus) e a mangueira (Mangifera indica). 
Outro ponto ressaltado é a existência de espécies vegetais que se destacam como recurso alimentar significativo para a fauna, dentre os quais a maria-fernanda (Posoqueria latifolia), a copaíba (Copaifera langsdorfii), o andá-açú (Joannesia princeps), bem como espécies dos gêneros Piper, Solanum e Ficus (Oliveira et al. 1995).

\section{MATERIAL E MÉTODOS}

No período de março de 1994 a maio de 1998, realizaram-se 45 noites de coleta (uma em cada mês) na área do Parque Estadual da Pedra Branca, com o auxílio de redes de espera ("mist-nets") de 6,9 e $12 \mathrm{~m}$ de comprimento por $2 \mathrm{~m}$ de altura, armadas ao nível do solo, em trilhas, próximo a possíveis fontes de alimento (vegetais em floração ou frutificação) ou locais de abrigo. Em cada sessão de captura, quatro a seis redes foram estendidas antes do anoitecer e retiradas às 24:00 h, sendo vistoriadas em intervalos de 15 minutos ou menos, dependendo da atividade dos morcegos.

Os morcegos capturados foram identificados no campo e para cada animal capturado foi obtido o comprimento do antebraço com paquímetro de precisão $0,05 \mathrm{~mm}$.

Caso estivessem em período reprodutivo, os animais eram soltos ao final da coleta, após identificação e anotação dos dados pertinentes. Os animais levados para o laboratório foram sacrificados com éter etílico, e conservados em álcool a $70^{\circ} \mathrm{GL}$, após fixação em formol $10 \%$, ou preparados sob a forma de pele cheia, após taxidermia, e incorporados à Coleção Adriano Lúcio Peracchi (ALP), atualmente depositada no Instituto de Biologia da Universidade Federal Rural do Rio de Janeiro (UFRRJ).

Para 23 espécies estudadas, são apresentadas, em milímetros (mm), a medida de antebraço e 13 medidas cranianas, efetuadas segundo critérios adotados por VIZOTTO \& TADDEI (1973). Para a medida do comprimento do antebraço, foram considerados os valores obtidos dos exemplares preservados em álcool ou taxidermizados, tomados do lado direito do corpo. As medidas, cujas abreviaturas aparecem nas tabelas e no decorrer do texto, são as seguintes: (AB) comprimento do antebraço, (B) comprimento basal, (Cb) comprimento côndilo-basal, (Cm) comprimento da mandíbula, (CM-I) comprimento da série de dentes inferiores, (CM-S) comprimento da série de dentes superiores, (Cpt) comprimento palatal, (CT) comprimento total do crânio, (Lc) largura entre os caninos superiores, (Lcx) largura da caixa craniana, (Lm) largura externa dos molares, (Lmt) largura mastóide, (Lpo) largura da constrição pós-orbital, (Lz) largura zigomática.

Para cada medida são fornecidos a média e os valores mínimos e máximos (para $\mathrm{N}=2$ ). As medidas de machos e fêmeas foram tratadas separadamente. Os dados morfométricos apresentados incluem apenas medidas de exemplares adultos, com epífises ossificadas.

A organização sistemática e a nomenclatura das espécies, bem como a ordem de citação das mesmas, seguem KOOPMAN (1993), a menos que seja indicado no decorrer do texto. 


\section{RESULTADOS E DISCUSSÃO}

Os trabalhos de campo permitiram a captura de 681 exemplares de 24 espécies, pertencentes a três famílias: Phyllostomidae (21 espécies), Vespertilionidae (duas espécies) e Molossidae (uma espécie) (Tab. I). Desse total, foram analisados 150 exemplares de 23 espécies.

Tabela I. Espécies de morcegos capturados no Parque Estadual da Pedra Branca (Rio de Janeiro), número de indivíduos (N), freqüência de captura em redes de espera (\%) e o número de machos e fêmeas de cada espécie incorporados à Coleção Adriano Lúcio Peracchi, UFRRJ (NALP).

\begin{tabular}{|c|c|c|c|c|}
\hline \multirow{2}{*}{ Espécies } & \multirow{2}{*}{$\mathrm{N}$} & \multirow{2}{*}{$\%$} & \multicolumn{2}{|c|}{$N_{\text {ALP }}$} \\
\hline & & & Machos & Fêmeas \\
\hline \multicolumn{5}{|l|}{ Phyllostomidae } \\
\hline Chrotopterus auritus (Peters, 1856) & 3 & 0,004 & 2 & - \\
\hline Micronycteris megalotis (Gray, 1842) & 1 & 0,001 & 1 & - \\
\hline Micronycteris minuta (Gervais, 1856) & 1 & 0,001 & 1 & - \\
\hline Mimon bennettii (Gray, 1838) & 1 & 0,001 & - & - \\
\hline Phyllostomus hastatus (Pallas, 1767) & 6 & 0,009 & 4 & 1 \\
\hline Tonatia bidens (Spix, 1823) & 2 & 0,003 & 1 & 1 \\
\hline Lonchophylla bokermanni Sazima, Vizzoto \& Taddei, 1978 & 1 & 0,001 & - & 1 \\
\hline Lonchophylla mordax Thomas, 1903 & 2 & 0,003 & 1 & 1 \\
\hline Anoura caudifera (E. Geoffroy, 1818) & 11 & 0,016 & 4 & 4 \\
\hline Glossophaga soricina (Pallas, 1766) & 17 & 0,025 & 4 & 5 \\
\hline Carollia perspicillata (Linnaeus, 1758) & 101 & 0,148 & 10 & 6 \\
\hline Artibeus fimbriatus Gray, 1838 & 139 & 0,206 & 18 & 14 \\
\hline Artibeus lituratus (Olfers, 1818) & 265 & 0,390 & 6 & 10 \\
\hline Artibeus obscurus Schinz, 1821 & 20 & 0,030 & 8 & 2 \\
\hline Chiroderma doriae Thomas, 1891 & 5 & 0,007 & 2 & 3 \\
\hline Platyrrhinus lineatus (E. Geoffroy, 1810) & 5 & 0,007 & 1 & 3 \\
\hline Platyrrhinus recifinus (Thomas, 1901) & 2 & 0,003 & 2 & - \\
\hline Sturnira lilium (E. Geoffroy, 1810) & 27 & 0,040 & 5 & 9 \\
\hline Vampyressa pusilla (Wagner, 1843) & 7 & 0,010 & 5 & - \\
\hline Desmodus rotundus (E. Geoffroy, 1810) & 41 & 0,060 & 2 & - \\
\hline Diphylla ecaudata Spix, 1823 & 4 & 0,006 & 4 & - \\
\hline \multicolumn{5}{|l|}{ Vespertilionidae } \\
\hline Eptesicus brasiliensis (Desmarest, 1819) & 1 & 0,001 & - & 1 \\
\hline Myotis nigricans (Schinz, 1821) & 11 & 0,016 & 3 & 2 \\
\hline \multicolumn{5}{|l|}{ Molossidae } \\
\hline Molossus molossus (Pallas, 1766) & 8 & 0,012 & 1 & 2 \\
\hline Total & 681 & 1,000 & 85 & 65 \\
\hline
\end{tabular}

Cabe ressaltar que esses dados não são uma representação verdadeira de toda a quiropterofauna do Parque, uma vez que a metodologia de captura empregada limitou-se ao uso de redes de espera próximo a vegetais em floração e frutificação. SIMMONS \& VOSS (1998) mencionam que algumas espécies são difíceis de capturar mediante redes de espera, em especial filostomíneos, vespertilionídeos e molossídeos e recomendam o uso de métodos adicionais de captura em complementação às redes, a fim de aumentar a eficiência do inventário. Coletas adicionais associadas ao emprego de métodos diversificados de captura poderão resultar em maior número de indivíduos e espécies, especialmente de Vespertilionidae e Molossidae. 
As observações sobre as espécies de morcegos inventariadas são apresentadas a seguir.

\section{Phyllostomidae Gray, 1825 \\ Phyllostominae Gray, 1825}

\section{Chrotopterus auritus (Peters, 1856)}

Localidade tipo: México, modificado para o Brasil, Santa Catarina, por CARTER \& DOLAN (1978).

Distribuição geográfica: Veracruz (México) até as Guianas; sul e sudeste do Brasil e norte da Argentina (KoOPMAN 1993).

Total de exemplares capturados: 03 .

Material examinado (02): macho ALP 5701; macho ALP 5854.

Descrições e dados morfométricos para essa espécie, embora baseados em poucos exemplares, foram fornecidos por autores como LIMA (1926), VIEIRA (1942), TAdDEI (1975a), SWANEPOEL \& GenOWAyS (1979), Williams \& GENOWAYS (1980) e MEDELLÍN (1989).

As dimensões encontradas (Tab. II) de modo geral encontram-se dentro da variação citada por MEDELLín (1989). Também não há diferenciação em relação à variação fornecida por TADDEI (1975a), para o Estado de São Paulo. As medidas também estão dentro da variação encontrada por SWANEPOEL \& GENOWAYS (1979), para exemplares do México e América Central.

\section{Micronycteris megalotis (Gray, 1842)}

Localidade tipo: Brasil, São Paulo, Perequê.

Distribuição geográfica: Tamaulipas e Jalisco (México) até Peru, Bolívia e Brasil; Trinidad e Tobago; Ilha Margarita (Venezuela); Granada (KoOPMAN 1993).

Total de exemplares capturados: 01 .

Material examinado (01): macho ALP 5679, cujo crânio estava danificado na parte basal posterior e apresentava arcos zigomáticos partidos em ambos os lados. As medidas desse exemplar são apresentadas na tabela II.

Entre os caracteres empregados para diferenciar Micronycteris megalotis (Gray, 1842) de Micronycteris minuta (Gervais, 1856), ambas incluídas no "subgênero Micronycteris" (SANBORN 1949) estão calcâneo mais longo que o pé, banda interauricular com um entalhe baixo no centro, pré-molares superiores subiguais em altura e segundo pré-molar inferior não muito reduzido em relação ao primeiro e terceiro (SANBORN 1949; ALONSO-MEJía \& MEDELlín 1991; GENOWAyS \& Williams 1986; SimMONS 1996). Coloração ventral marrom e comprimento da segunda falange do metacarpo IV mais curta que a primeira também têm sido referidos como diagnósticos para M. megalotis por SimMONS (1996).

O exemplar colecionado no PEPB foi atribuído a $M$. megalotis, com base nos caracteres propostos pelos autores mencionados acima.

ANDERSON et al. (1982) mencionaram que os exemplares de M. megalotis, na Bolívia, são distinguidos de $M$. minuta por inclinação mais abrupta da fronte, 
caixa craniana mais expandida dorsalmente e região interorbital mais estreita que em M. minuta. Contudo, esses caracteres não foram observados no exemplar de $M$. megalotis, nem no exemplar atribuído a M. minuta, em especial no que diz respeito à largura da região interorbital, para a qual os dois exemplares apresentaram valores muito próximos (ver Tab. II).

\section{Micronycteris minuta (Gervais, 1856)}

Localidade tipo: Brasil, Bahia, Capela Nova.

Distribuição geográfica: Nicarágua ao sul do Brasil; Peru; Guianas; Trinidad; Bolívia (KOOPMAN 1993).

Total de exemplares capturados: 01.

Material examinado (01): macho ALP 5813.

Medidas obtidas para o macho colecionado no PEPB são apresentadas na tabela II.

O exemplar colecionado do PEPB pôde ser identificado como $M$. minuta, utilizando-se os caracteres morfológicos propostos pelos autores citados na discussão sobre $M$. megalotis, especialmente a presença de um entalhe profundo e bem distinto. O exemplar também possui largura mastóide maior que a largura zigomática, um caracter que, segundo Simmons (1996), também separa M. minuta de $M$. megalotis.

Tabela II. Medidas de exemplares de Chrotopterus auritus, Micronycteris megalotis e Micronycteris minuta do Parque Estadual da Pedra Branca, Rio de Janeiro. (N) Número de exemplares.

\begin{tabular}{|c|c|c|c|c|c|}
\hline \multirow{3}{*}{ Caracteres } & \multicolumn{3}{|c|}{ Chrotopterus auritus } & \multirow{3}{*}{$\frac{\text { Micronycteris megalotis }}{\text { Machos }}$} & \multirow{3}{*}{$\begin{array}{c}\text { Micronycteris minuta } \\
\text { Machos }\end{array}$} \\
\hline & \multicolumn{3}{|c|}{ Machos } & & \\
\hline & Média & Mínimo-Máximo & N & & \\
\hline$A B$ & 84,20 & $84,0-84,4$ & 2 & 34,5 & 35,5 \\
\hline CT & 37,15 & $36,8-37,5$ & 2 & 18,1 & 19,0 \\
\hline $\mathrm{Cb}$ & 32,80 & $32,4-33,2$ & 2 & - & 16,6 \\
\hline B & 29,40 & $28,8-30,0$ & 2 & - & - \\
\hline Cpt & - & - & & 8,5 & - \\
\hline CM-S & 14.05 & $14,0-14,1$ & 2 & 6,8 & 6,8 \\
\hline CM-I & 15,80 & $15,5-16,1$ & 2 & 7,2 & 7,2 \\
\hline Lm & 12,50 & $12,0-13,0$ & 2 & 5,7 & 5,4 \\
\hline $\mathrm{Cm}$ & 25,45 & $25,3-25,6$ & 2 & 11,3 & 11,6 \\
\hline LC & 8,60 & $8,5-8,7$ & 2 & 3,2 & 3,2 \\
\hline Lpo & 6,20 & $6,0-6,4$ & 2 & 4,1 & 4,2 \\
\hline Lz & 19,75 & $19,5-20,0$ & 2 & - & 8,2 \\
\hline LCx & 13,85 & $13,7-14,0$ & 2 & 7,2 & 7,7 \\
\hline Lmt & 17,90 & $17,5-18,3$ & 2 & - & 8,7 \\
\hline
\end{tabular}

\section{Mimon bennettii (Gray, 1838)}

Localidade tipo: Brasil, São Paulo, Ipanema.

Distribuição geográfica: Sul do México até a Colômbia; Guianas; sudeste do Brasil (KoOPMAN 1993).

Total de exemplares capturados: 01 fêmea grávida, capturada e solta em 30-VI-1994. 


\section{Phyllostomus hastatus (Pallas, 1767)}

Localidade tipo: Suriname

Distribuição geográfica: Honduras às Guianas, leste do Brasil, Paraguai, norte da Argentina e Peru; Trinidad e Tobago; Ilha Margarita (Venezuela); Bolívia (KOOPMAN 1993).

Total de exemplares capturados: 06.

Material examinado (05): macho ALP 5678; macho ALP 5775; macho ALP 5776; macho ALP 5786; fêmea ALP 5785.

Os dados morfométricos obtidos para essa espécie encontram-se na tabela III. As médias dos machos são maiores do que as médias fornecidas por TADDEI (1975 a), para material de São Paulo, e por WiLlig (1983), para exemplares da Caatinga em Pernambuco e do Cerrado, no Ceará.

\section{Tonatia bidens (Von Spix, 1823)}

Localidade tipo: Brasil, Bahia, Rio São Francisco.

Distribuição geográfica: Chiapas (México) e Belize ao norte da Argentina, Paraguai e Brasil; Trinidad (KoOPMAN 1993). Após a revisão do "complexo Tonatia bidens" por Williams et al. (1995), foram reconhecidas duas espécies distintas: Tonatia bidens (Von Spix, 1823) com distribuição limitada ao leste e sul do Brasil, Paraguai e norte da Argentina, e Tonatia saurophilla Koopman \& Williams, 1951, distribuída pela América Central, norte e oeste da América do Sul e Jamaica.

Total de exemplares capturados: 02.

Material examinado (02): macho ALP 5715; fêmea ALP 5826.

Dados morfométricos são apresentados na tabela III.

Tabela III. Medidas de exemplares de Phyllostomus hastatus e Tonatia bidens do Parque Estadual da Pedra Branca, Rio de Janeiro. (N) Número de exemplares.

\begin{tabular}{|c|c|c|c|c|c|c|}
\hline \multirow{3}{*}{ Caracteres } & \multicolumn{4}{|c|}{ Phyllostomus hastatus } & \multicolumn{2}{|c|}{ Tonatia bidens } \\
\hline & \multicolumn{3}{|c|}{ Machos } & \multirow[t]{2}{*}{ Fêmeas } & \multirow[t]{2}{*}{ Machos } & \multirow[t]{2}{*}{ Fêmeas } \\
\hline & Média & Minimo-Máximo & $\mathrm{N}$ & & & \\
\hline$A B$ & 90,05 & $88,5-91,9$ & 4 & 90,0 & 50,8 & 51,0 \\
\hline CT & 41,95 & $41,6-44,3$ & 4 & 40,0 & 26,6 & 26,9 \\
\hline $\mathrm{Cb}$ & 36,38 & $36,6-36,9$ & 4 & 35,5 & 23,2 & 22,8 \\
\hline B & 32,85 & $32,7-33,4$ & 4 & 31,9 & 20,7 & 20,2 \\
\hline Cpt & 18,73 & $18,8-19,2$ & 4 & 19,0 & 12,2 & 12,0 \\
\hline CM-S & 14,05 & $14,0-14,4$ & 4 & 13,9 & 9,1 & 9,3 \\
\hline CM-I & 16,08 & $16,0-16,5$ & 4 & 15,0 & 10,2 & 10,2 \\
\hline $\operatorname{Lm}$ & 13,58 & $13,3-13,9$ & 4 & 13,0 & 7,6 & 7,7 \\
\hline $\mathrm{Cm}$ & 26,98 & $27,3-27,5$ & 4 & 26,4 & 16,6 & 16,4 \\
\hline Lc & 10,20 & $10,0-10,5$ & 4 & 9,4 & 5,3 & 5,4 \\
\hline Lpo & 7,70 & $7,4-8,0$ & 4 & 7,4 & 5,7 & 6,0 \\
\hline $\mathrm{Lz}$ & 22,38 & $22,2-22,8$ & 4 & 21,4 & 12,7 & 13,0 \\
\hline LCx & 15,18 & $14,9-15,5$ & 4 & 14,4 & 10,7 & 11,1 \\
\hline Lmt & 21,08 & $20,9-21,4$ & 4 & 19,6 & 12,6 & 12,6 \\
\hline
\end{tabular}

Até a revisão de WiLliams et al. (1995), as formas referidas a "Tonatia bidens" diferenciavam-se dos espécimens de Tonatia sivicola (D’Orbigny, 1836) e Tonatia evotis Davis \& Carter, 1978 pelas orelhas menores que $32 \mathrm{~mm}$, largura 
ao nível da constrição pós-orbital maior que 5,0 $\mathrm{mm}$ e ausência de banda interauricular sobre a fronte (GOODWIN 1942; DAVIS \& CARTER 1978), além de uma faixa branca sobre o topo da cabeça, (GENOWAYS \& Williams 1984; MEDELLÍN \& ARITA 1989).

Segundo Williams et al. (1995), T. bidens diferencia-se de T. saurophila pela ausência de processo secundário sobre o mastóide, incisivos inferiores estreitos, caninos inferiores quase em contato na margem medial posterior, coroa do segundo pré-molar inferior obscurecida pelos pré-molares adjacentes, além da ausência de uma faixa branca sobre o topo da cabeça. Os exemplares examinados puderam ser identificados como $T$. bidens com base em todos os caracteres propostos pelos autores acima para a espécie. Houve sobreposição da maioria das medidas aqui obtidas com aquelas reportadas para $T$. silvicola e $T$. saurophila, espécies com porte comparável a T. bidens ( $c f$. GOODWIN 1942; DAVIS \& CARTER 1978; GENOWAYS \& Williams 1984; MEdellín \& ARITA 1989; Williams et al. 1995).

\section{Lonchophyllinae Griffiths, 1982}

\section{Lonchophylla bokermanni Sazima, Vizotto \& Taddei, 1978}

Localidade tipo: Brasil, Minas Gerais, Jaboticatubas, Serra do Cipó.

Distribuição geográfica: Sudeste do Brasil (KoOPMAN 1993). Recentemente, Lonchophylla bokermanni teve sua distribuição ampliada por BAPTISTA \& OLIVEIRA (1998), através de registros para o Brasil Central (Alto Tocantins, Goiás) e para o Nordeste (Cocorobó, Bahia).

Total de exemplares capturados: 01 .

Material examinado (01): fêmea ALP 5820.

Medidas obtidas para a fêmea colecionada no PEPB são apresentadas na tabela IV.

Segundo SAZIMA et al. (1978), Lonchophylla bokermanni pode ser distinguida de Lonchophylla mordax Thomas, 1903, com a qual ocorre em simpatria no leste do Brasil pelos seguintes caracteres: AB superior a $38,7 \mathrm{~mm}, \mathrm{CM}-\mathrm{S}$ entre 7,8 e $8,6 \mathrm{~mm}$, incisivos superiores trilobulados, segundo pré-molar superior com lobo interno reduzido e raiz da face lingual distintamente posterior ao meio do dente e maiores dimensões craniais, especialmente $\mathrm{CT}, \mathrm{Cb}, \mathrm{B}$ e Cm. Lonchophylla dekeyseri Taddei, Vizotto \& Sazima, $1983(\mathrm{AB}=34,7$ a $37,7 \mathrm{~mm}$ e $\mathrm{CT}=22,0$ a 22,6 $\mathrm{mm}$ ) é menor que L. bokermanni (TADDEI et al. 1983).

Um dos três indivíduos de Lonchophylla amostrados pelo presente estudo foi identificado como L. bokermanni, mas alguns caracteres usualmente referidos como diagnósticos (SAZIMA et al. 1978; TADDEI et al. 1983) mostraram-se controversos.

O exemplar em estudo apresentou alguns caracteres dentários que divergiram da descrição de SAZIMA et al. (1978): incisivos inferiores internos bilobulados; segundo pré-molar inferior separado, em ambos os lados da arcada, do primeiro e do terceiro por um espaço grande (não pequeno) e coroa do terceiro pré-molar inferior distintamente separada do primeiro molar por um espaço grande. 
As informações a respeito da morfometria dessa espécie limitam-se à descrição da espécie por SAZIMA et al. (1978) e ao trabalho de TADDEI et al. (1988) sobre amostra de cinco exemplares coligidos da Ilha Grande, no Estado do Rio de Janeiro. As dimensões cranianas do exemplar em estudo estão dentro da variação referente à série-tipo (SAZIMA et al. 1978) e dos extremos encontrados por TADDEI et al. (1988) (Tab. II), com exceção do AB, muito menor que as medidas dos exemplares da série-tipo, superpondo-se neste caráter a L. mordax. No entanto, o antebraço se aproxima do mínimo encontrado para as fêmeas de L. bokermanni da Ilha Grande (Tab. IV).

Tabela IV. Medidas de exemplares de Lonchophylla bokermanni (extremos) fornecidas por SaZIMA et al. (1978), para a série-tipo (Serra do Cipó, Jaboticatubas, Minas Gerais) ${ }^{1}$, para o presente estudo ${ }^{2}$, e por TADDEI et al. (1988), para exemplares da llha Grande (Rio de Janeiro) ${ }^{3}$, além de medidas de exemplares de Lonchophylla mordax do Parque Estadual da Ilha Grande.

\begin{tabular}{|c|c|c|c|c|c|c|c|}
\hline \multirow{3}{*}{ Caracteres } & \multicolumn{5}{|c|}{ Lonchophylla bokermanni* } & \multirow{2}{*}{\multicolumn{2}{|c|}{$\begin{array}{c}\text { Lonchophylla mordax } \\
\text { PEPB }\end{array}$}} \\
\hline & \multicolumn{2}{|c|}{ Série-tipo $(M G)^{1}$} & \multirow{2}{*}{$\frac{\text { PEPB }^{2}}{\text { Fêmea }}$} & \multicolumn{2}{|c|}{ Ilha Grande $(R J)^{3}$} & & \\
\hline & Machos & Fêmeas & & Machos & Fêmeas & Machos & Fêmeas \\
\hline$A B$ & $38,7-40,0$ & $40,0-41,3$ & 35,5 & $36,7-36,8$ & $35,9-36,3$ & 35,1 & 35,8 \\
\hline CT & $25,2-25,3$ & $25,4-26,3$ & 25,3 & 25,0 & $24,8-25,8$ & 24,0 & 23,8 \\
\hline $\mathrm{Cb}$ & $23,9-24,1$ & $24,0-25,0$ & 24,1 & 24,0 & $23,7-24,7$ & 23,0 & 22,7 \\
\hline B & $22,0-22,2$ & $21,9-22,9$ & 22,7 & 22,0 & $21,7-22,7$ & 21.0 & 20,2 \\
\hline CM-S & $8,0-8,2$ & $7,8-8,6$ & 8,2 & 8,2 & $8,0-8,1$ & 7,7 & 7,8 \\
\hline $\mathrm{CM}-1$ & $8,4-8,7$ & $8,2-8,9$ & 8,5 & 8,6 & $8,4-8,5$ & 8,0 & 8,2 \\
\hline $\mathrm{Lm}$ & $5,3-5,7$ & $5,2-5,7$ & 5,4 & 5,3 & $5,1-5,2$ & 5,2 & 5,1 \\
\hline $\mathrm{Cm}$ & $17,3-17,4$ & $17,4-18,0$ & 17,2 & 17,3 & $16,8-17,7$ & 16,0 & 16,1 \\
\hline Lc & $4,1-4,2$ & $3,7-4,0$ & 3,8 & - & $4,0-4,1$ & 4,1 & 3,6 \\
\hline Lpo & $4,8-4,9$ & $4,8-5,0$ & 4,9 & 4,6 & $4,6-4,7$ & 5,0 & 4,6 \\
\hline LCx & $9,2-9,5$ & $9,3-9,7$ & 8,6 & 9,2 & $9,1-9,3$ & 8,5 & 8,5 \\
\hline $\mathrm{Lmt}$ & $9,4-9,7$ & $9,4-9,6$ & 9,1 & 9,4 & $9,2-9,4$ & 9,0 & 8,9 \\
\hline
\end{tabular}

( $\left.{ }^{\star}\right)$ Lonchophylla bokermanni: $\left({ }^{1} N\right) 2$ machos e 7 fêmeas, $\left({ }^{2} N\right) 1$ fêmea, $\left({ }^{3} N\right) 1$ macho e 3 fêmeas.

\section{Lonchophylla mordax Thomas, 1903}

Localidade tipo: Brasil, Bahia, Lamarão.

Distribuição geográfica: Costa Rica ao Equador; leste do Brasil; provavelmente Peru e Bolívia (KOOPMAN 1993). Antes aparentemente restrita ao Nordeste (TADDEI et al. 1988), essa espécie teve sua ocorrência confirmada na região Sudeste por PEDro \& PASSOS (1995) para o Estado do Espírito Santo e EsBERÁRD (1998) para o Rio de Janeiro.

Total de exemplares capturados: 02 .

Material examinado (02): macho ALP 5860; fêmea ALP 5664.

Medidas obtidas para exemplares do PEPB são apresentadas na tabela IV. Dois exemplares do PEPB referidos a Lonchophylla foram identificados como $L$. mordax segundo os critérios propostos por SAZIMA et al. (1978) e TADDEI et al. (1983). Os comprimentos de antebraço também estão dentro dos extremos propostos por SANBORN (1941). 
Os indivíduos do PEPB possuem o segundo pré-molar estreito, sem o desenvolvimento de cúspide ou lobo interno, um caráter usualmente proposto como diagnóstico para L. mordax em relação a L. bokermanni.

Segundo KOOPMAN (1994), L. mordax e L. bokermanni não podem ser separadas com base nesta característica, sendo as espécies separadas pelo tamanho: L. mordax menor $(\mathrm{AB}=32$ a $37 \mathrm{~mm}, \mathrm{Cb}=20$ a $23 \mathrm{~mm}$ ) e L. bokermanni maior ( $\mathrm{AB}$ $=38$ a $42 \mathrm{~mm}, \mathrm{Cb}=23$ a $25 \mathrm{~mm}$ ).

Os estudos morfométricos, sobre amostragem representativa para essa espécie, são escassos. WILLIG (1983) forneceu medidas para 72 exemplares de L. mordax procedentes do Nordeste do Brasil. Medidas de exemplares dessa espécie para o Sudeste brasileiro foram fornecidas por RUSCHI (1953). A identificação de um morcego do Espírito Santo por esse autor parece duvidosa, conforme discutido por PINE \& RUSCHI (1976).

\section{Glossophaginae Bonaparte, 1845}

\section{Anoura caudifera (E. Geoffroy, 1818)}

Localidade tipo: Brasil, Rio de Janeiro.

Distribuição geográfica: Colômbia, Venezuela, Guianas, Brasil, Equador, Peru, Bolívia, noroeste da Argentina (KoOPMAN 1993).

Total de exemplares capturados: 11 .

Material examinado (08): macho ALP 5665; macho ALP 5743; macho ALP 5780; macho ALP 5800; fêmea ALP 5666; fêmea ALP 5788; fêmea ALP 5805; fêmea ALP 5864.

Os dados morfométricos obtidos para Anoura caudifera encontram-se na tabela V.

SANBORN (1933 apud TADDEI 1975b) tratou Anoura Gray, 1838 e Lonchoglossa Peters, 1868 como gêneros distintos. Esse arranjo foi seguido por HUSSON (1962) e VieIRA (1942). TAMSITT \& VALDIVIESO (1966) em concordância com CABRERA (1958) consideraram esses táxons congenéricos, diferenciados apenas pela ausência de cauda em Anoura e a presença desta em Lonchoglossa. Em estudo sobre material do Suriname, Husson (1962) comentou que a cauda era muito curta, às vezes imperfeitamente ossificada, escondida na base da membrana interfemural, fato posteriormente observado por WiLLIAMS \& GENOWAYS (1980), também em amostras do Suriname. Todos os exemplares do PEPB possuíam cauda distinta, embora com comprimento bastante variável.

A denominação do epíteto específico "caudifera" segue HANDLEY (1984).

\section{Glossophaga soricina (Pallas, 1766)}

Localidade tipo: Suriname. Segundo Alvarez et al. (1991: 5), "a localidade tipo de Vespertilio soricinus é desconhecida, mas Pallas examinou exemplares do Suriname e Ilhas do Caribe, e foi restrita para o 'norte da América do Sul' por Rehn (1902) e depois ao Suriname por Miller (1912)".

Distribuição geográfica: Tamaulipas, Sonora e Ilhas Três Marias (México) às Guianas; sudeste do Brasil, norte da Argentina e Peru; Ilha Margarita (Venezuela); Trinidad; Granada (Pequenas Antilhas); Jamaica; talvez Bahamas (KoOPMAN 1993). 
Total de exemplares capturados: 17.

Material examinado (09): macho ALP 5702; macho ALP 5732; macho ALP 5790; macho ALP 5863; fêmea ALP 5723; fêmea ALP 5726; fêmea ALP 5787; fêmea ALP 5818; fêmea ALP 5819.

Os dados morfométricos para essa espécie são apresentados na tabela V.

Tabela V. Medidas de exemplares de Anoura caudifera e Glossophaga soricina do Parque Estadual da Pedra Branca, Rio de Janeiro. (N) Número de exemplares.

\begin{tabular}{|c|c|c|c|c|c|c|c|c|c|c|c|c|}
\hline \multirow{3}{*}{ Caracteres } & \multicolumn{6}{|c|}{ Anoura caudifera } & \multicolumn{6}{|c|}{ Glossophaga soricina } \\
\hline & \multicolumn{3}{|c|}{ Machos } & \multicolumn{3}{|c|}{ Fêmeas } & \multicolumn{3}{|c|}{ Machos } & \multicolumn{3}{|c|}{ Fêmeas } \\
\hline & Média & Min-Máx & $\mathrm{N}$ & Média & Min-Máx & $\mathrm{N}$ & Média & Min-Máx & $\mathrm{N}$ & Média & Mín-Máx & $\mathrm{N}$ \\
\hline$A B$ & 26,23 & $35,5-37,0$ & 4 & 35,80 & $35,3-36,4$ & 3 & 35,13 & $34,6-36,0$ & 3 & 36,08 & $33,3-37,2$ & 5 \\
\hline CT & 22,75 & $22,6-23,1$ & 4 & 22,80 & $22,5-23,1$ & 3 & 20,80 & $20,5-21,0$ & 3 & 21,28 & $20,7-21,7$ & 5 \\
\hline $\mathrm{Cb}$ & 22,00 & $21,6-22,5$ & 4 & 22,10 & $21,9-22,3$ & 3 & 19,50 & $19,3-19,7$ & 3 & 20.08 & $19,6-20,6$ & 5 \\
\hline B & 19,98 & $19,8-20,1$ & 4 & 19,90 & $19,7-20,0$ & 3 & 17,55 & $17,5-17,6$ & 2 & 17,92 & $17,5-18,4$ & 5 \\
\hline $\mathrm{Cpt}$ & 12,23 & $12,0-12,4$ & 4 & 12,30 & $11,9-12,8$ & 3 & 11,40 & $11,4-$ & 1 & 11,52 & $10,8-12,0$ & 5 \\
\hline CM-S & 8,38 & $8,3-8,6$ & 4 & 8,40 & $8,1-8,7$ & 3 & 7,07 & $6,8-7,3$ & 3 & 7,30 & $7,2-7,5$ & 5 \\
\hline $\mathrm{CM}-1$ & 8,75 & $8,6-8,9$ & 4 & 8,67 & $8,3-9,0$ & 3 & 7,53 & $7.3-7.7$ & 3 & 7.66 & $7,4-7,8$ & 5 \\
\hline $\mathrm{Lm}$ & 4,90 & $4,1-5,2$ & 4 & 5,13 & $5,0-5,3$ & 3 & 5,23 & $5,0-5,7$ & 3 & 5,44 & $5,2-5,5$ & 5 \\
\hline $\mathrm{Cm}$ & 16,20 & $15,7-16,7$ & 4 & 16,27 & $16,1-16,4$ & 3 & 13,50 & $13,4-13,6$ & 3 & 14,12 & $14,0-14,3$ & 5 \\
\hline Lc & 4,00 & $3,6-4,2$ & 4 & 3,93 & $3,7-4,1$ & 3 & 3,77 & $3,6-3,9$ & 3 & 4,82 & $3,5-4,1$ & 5 \\
\hline Lpo & 4,55 & $4,0-4,8$ & 4 & 4,63 & $4,6-4,7$ & 3 & 4,67 & $4,5-4,9$ & 3 & 4,84 & $4,6-5,1$ & 5 \\
\hline $\mathrm{Lz}$ & 9,35 & $8,8-9,8$ & 4 & 9,30 & $9,2-9,4$ & 3 & 8,60 & $8,6-$ & 1 & 9,26 & $8,9-9,5$ & 5 \\
\hline LCx & 8,63 & $8,1-8,9$ & 4 & 8,53 & $8,4-8,6$ & 3 & 8,13 & $8,0-8,3$ & 3 & 8,42 & $8,3-8,6$ & 5 \\
\hline Lmt & 9,30 & $8,9-9,5$ & 4 & 9,20 & $8,8-9,5$ & 3 & 8,60 & $8,3-9,0$ & 3 & 8,86 & $8,5-9,2$ & 5 \\
\hline
\end{tabular}

Atualmente a melhor referência para $G$. soricina é a revisão de WEBSTER (1993), que incluiu medidas comparativas detalhadas para essa espécie. Este autor encontrou variação clinal nas dimensões externas e cranianas, em que as populações da maior parte da América do Sul são em geral menores que a média.

\section{Carollinae Miller, 1924}

\section{Carollia perspicillata (Linnaeus, 1758)}

Localidade tipo: Suriname.

Distribuição geográfica: Oaxaca, Veracruz e Yucatán (México) ao Peru, Bolívia, Paraguai, Brasil e Guianas; Trinidad e Tobago; Granada (Pequenas Antilhas); provavelmente Jamaica (KoOPMAN 1993).

Total de exemplares capturados: 101.

Material examinado (16): macho ALP 5660; macho ALP 5667; macho ALP 5668; macho ALP 5669; macho ALP 5747; macho ALP 5755; macho ALP 5756; macho ALP 5801; macho ALP 5822; macho ALP 5857; fêmea ALP 5661; fêmea ALP 5672; fêmea ALP 5676; fêmea ALP 5680; fêmea ALP 5814; fêmea ALP 5855.

Os dados morfométricos obtidos para essa espécie são apresentados na tabela VI.

Revta bras. Zool. 19 (Supl. 2): 113 - 140, 2002 
Tabela VI. Medidas de exemplares de Carollia perspicillata do Parque Estadual da Pedra Branca, Rio de Janeiro. (N) Número de exemplares.

\begin{tabular}{|c|c|c|c|c|c|c|}
\hline \multirow{2}{*}{ Caracteres } & \multicolumn{3}{|c|}{ Machos } & \multicolumn{3}{|c|}{ Fêmeas } \\
\hline & Média & Mínimo-Máximo & $\mathrm{N}$ & Média & Mínimo-Máximo & N \\
\hline$A B$ & 40,14 & $38,2-41,5$ & 7 & 40,34 & $38,5-42,2$ & 5 \\
\hline CT & 22,60 & $21,9-23,5$ & 7 & 22,18 & $21,9-22,8$ & 6 \\
\hline $\mathrm{Cb}$ & 20,40 & $19,5-21,4$ & 7 & 19,97 & $19,5-20,7$ & 6 \\
\hline B & 18,17 & $17,3-19,0$ & 7 & 17,65 & $17,2-18,4$ & 6 \\
\hline $\mathrm{Cpt}$ & 10,06 & $9,6-10,7$ & 7 & 9,73 & $9,1-10,5$ & 6 \\
\hline CM-S & 7,39 & $7,0-8,0$ & 7 & 7,32 & $7,0-7,5$ & 6 \\
\hline $\mathrm{CM}-1$ & 8,03 & $7,6-8,2$ & 7 & 7,97 & $7,6-8,2$ & 6 \\
\hline $\mathrm{Lm}$ & 7,49 & $7,2-7,9$ & 7 & 7,23 & $6,9-7,4$ & 6 \\
\hline $\mathrm{Cm}$ & 14,54 & $14,3-14,8$ & 7 & 14,18 & $13,9-14,6$ & 6 \\
\hline LC & 5,10 & $4,9-\quad 5,5$ & 7 & 4,95 & $4,6-5,2$ & 6 \\
\hline Lpo & 5,64 & $5,5-6,0$ & 7 & 5,42 & $5,1-5,6$ & 6 \\
\hline Lex & 9,47 & $9,0-9,8$ & 7 & 9,67 & $9,2-10,4$ & 6 \\
\hline $\mathrm{Lmt}$ & 11,11 & $10,7-11,9$ & 7 & 10,62 & $10,3-11,0$ & 6 \\
\hline
\end{tabular}

Conforme a revisão de PINE (1972), Carollia perspicillata (Linnaeus, 1758) distingue-se de Carollia brevicauda (Schinz, 1821) pelo maior tamanho, incisivos inferiores externos obscurecidos pelo cíngulos dos caninos, fileiras de dentes superiores retas, sem diástema entre os pré-molares e mandíbula em forma de $\mathrm{V}$, com séries de dentes inferiores mais longas. Além disso o autor mencionou que os pêlos de $C$. perspicillata são mais curtos que os de C. brevicauda e apresentam bandeamento menos distinto, mas ressalvou que indivíduos das populações da porção austral da distribuição geográfica apresentam pêlos longos e distintamente tricoloridos (banda basal escura e larga, contrastando fortemente com a banda central branca, seguida pela banda distal bem escura).

Um caráter apontado por MCLELLAN (1984) para distinção entre C. perspicillata e C. brevicauda diz respeito ao comprimento da série de dentes superiores, a primeira espécie com média de $7,53 \mathrm{~mm}$ e a segunda, com 6,95mm. As médias encontradas neste estudo para este parâmetro concordam com o valor proposto por esta autora para C. perspicillata.

Quanto aos incisivos inferiores, dentre 14 exemplares, oito apresentaram incisivos apenas parcialmente obscurecidos, quatro apresentavam incisivos perfeitamente visíveis e apenas 2 apresentaram os incisivos totalmente ocultados pelos cíngulos dos caninos.

Em todos os exemplares, notou-se que os pêlos dorsais são distintamente tricoloridos, com as bandas bem demarcadas umas das outras. Em geral, os exemplares puderam ser identificados como $C$. perspicillata, sem maiores problemas, segundo os critérios estabelecidos por PINE (1972) e MCLELLAN (1984)

Segundo Koopman (1982), C. brevicauda e C. perspicillata distribuem-se simpatricamente por vasta área da América do Sul, sendo que no Sudeste brasileiro, os registros da primeira espécie parecem limitar-se ao Estado do Espírito Santo onde se situa a localidade tipo e onde foi amostrada por PERACCHI \& ALBUQUERQUE (1993). Nos outros estados da região Sudeste, aparentemente apenas C. perspicillata tem sido registrada (PERACCHI \& ALBUQUERQUE 1971; 1986; TADDEI 1975 b; REIS et al. 1996; ESBERÁRD 1998). 


\section{Stenodermatinae Gervais, 1856}

\section{Artibeus fimbriatus Gray, 1838}

Localidade tipo: Brasil, Paraná, Serra do Mar, Morretes.

Distribuição geográfica: Do Nordeste passando pelo leste e sul do Brasil (Bahia a Santa Catarina) (Koopman 1993; MARques-Aguiar 1994), leste do Paraguai (HANDLEY 1989).

Total de exemplares capturados: 139.

Material examinado (32): macho ALP 5657; macho ALP 5674; macho ALP 5695; macho ALP 5712; macho ALP 5713; macho ALP 5737; macho ALP 5740; macho ALP 5751; macho ALP 5754; macho ALP 5757; macho ALP 5764; macho ALP 5778; macho ALP 5781; macho ALP 5798; macho ALP 5806; macho ALP 5824; macho ALP 5832; macho ALP 5833; fêmea ALP 5654; fêmea ALP 5673; fêmea ALP 5683; fêmea ALP 5698; fêmea ALP 5700; fêmea ALP 5704; fêmea ALP 5710; fêmea ALP 5741; fêmea ALP 5765; fêmea ALP 5769; fêmea ALP 5770; fêmea ALP 5779,; fêmea ALP 5802; fêmea ALP 5823.

Os dados morfométricos para Artibeus fimbriatus encontram-se na tabela VII.

Tabela VII. Medidas de exemplares de Artibeus fimbriatus e Artibeus lituratus do Parque Estadual da Pedra Branca, Rio de Janeiro. (N) Número de exemplares.

\begin{tabular}{|c|c|c|c|c|c|c|c|c|c|c|c|c|}
\hline \multirow{3}{*}{ Caracteres } & \multicolumn{6}{|c|}{ Artibeus fimbriatus } & \multicolumn{6}{|c|}{ Artibeus lituratus } \\
\hline & \multicolumn{3}{|c|}{ Machos } & \multicolumn{3}{|c|}{ Fêmeas } & \multicolumn{3}{|c|}{ Machos } & \multicolumn{3}{|c|}{ Fêmeas } \\
\hline & Média & Min-Máx & $N$ & Média & Min-Máx & $\mathrm{N}$ & Média & Min-Máx & $\mathrm{N}$ & Média & Mín-Máx & N \\
\hline$A B$ & 64,53 & $60,1-67,8$ & 14 & 65,61 & $62,8-68,0$ & 12 & 70,60 & $68,8-72,4$ & 5 & 70,39 & $68,0-72,7$ & 10 \\
\hline CT & 31,15 & $30,1-32,5$ & 14 & 31,20 & $30,3-31,9$ & 12 & 32,30 & $31,3-33,8$ & 5 & 32,23 & $31,0-33,3$ & 10 \\
\hline $\mathrm{Cb}$ & 28,15 & $27,3-29,5$ & 14 & 28,16 & $27,1-28,9$ & 12 & 28,78 & $27,3-30,0$ & 5 & 28,94 & $28,1-29,6$ & 10 \\
\hline B & 25,13 & $24,3-26,0$ & 14 & 25,08 & $24,4-26,0$ & 12 & 25,30 & $24,0-26,7$ & 5 & 25,56 & $24,4-26,1$ & 10 \\
\hline Cpt & 15,63 & $15,1-16,3$ & 14 & 15,54 & $14,8-16,1$ & 12 & 14,98 & $13,5-16,4$ & 5 & 15,59 & $14,8-16,2$ & 10 \\
\hline CM-S & 11,41 & $11,0-11,7$ & 14 & 11,36 & $10,9-11,7$ & 12 & 11,30 & $10,8-11,8$ & 5 & 11,52 & $11,0-11,9$ & 10 \\
\hline $\mathrm{CM}-\mathrm{I}$ & 12,64 & $12,3-12,9$ & 14 & 12,60 & $12,1-13,0$ & 12 & 12,76 & $12,2-13,3$ & 5 & 12,74 & $12,2-13,2$ & 10 \\
\hline Lm & 14,12 & $13,5-14,5$ & 14 & 14,06 & $13,3-14,7$ & 12 & 14,04 & $13,7-14,5$ & 5 & 13,88 & $13,4-14,2$ & 10 \\
\hline $\mathrm{Cm}$ & 20,86 & $19.6-21.7$ & 14 & 20.78 & $20,0-21,4$ & 12 & 21,26 & $20,1-21,8$ & 5 & 21,42 & $20,5-21,8$ & 10 \\
\hline LC & 9,06 & $8,7-9,5$ & 14 & 8,99 & $8,7-9,4$ & 12 & 9,00 & $8,3-9,6$ & 5 & 9,07 & $8,6-\quad 9,5$ & 10 \\
\hline Lpo & 7,60 & $7,1-8,1$ & 14 & 7,54 & $7,0-8,0$ & 12 & 7,28 & $7,0-8,0$ & 5 & 7,10 & $6,6-8,0$ & 10 \\
\hline Lz & 18,82 & $16,5-19,8$ & 14 & 18,88 & $17,9-19,7$ & 12 & 19,22 & $18,3-19,7$ & 5 & 19,48 & $19,0-20,3$ & 10 \\
\hline LCx & 13,68 & $13,3-14,4$ & 14 & 13,54 & $13,0-14,0$ & 12 & 14,32 & $13,6-14,8$ & 5 & 14,23 & $13,6-14,8$ & 10 \\
\hline Lmt & 16,48 & $15,1-17,2$ & 14 & 16,48 & $15,1-17,2$ & 12 & 17,24 & $16,5-17,8$ & 5 & 17,27 & $16,4-18,0$ & 10 \\
\hline
\end{tabular}

Artibeus fimbriatus, descrita por Gray em 1838 e ignorada por mais de 150 anos, foi redescoberta e redescrita por HANDLEY (1989). MYERS \& WETZEL (1979; 1983) forneceram a primeira citação moderna para a espécie, quando documentaram a sua possível ocorrência no Chaco paraguaio.

Externamente, A. fimbriatus é uma espécie de grande porte, muito semelhante a A. lituratus, da qual pode ser distinguida com facilidade, de acordo com HANDLEY (1989). O autor mencionou que A. fimbriatus possui pelagem mais longa e macia, coloração mais escura, listras faciais mais obsoletas ou indistintas e estreitas, além do 
ventre acinzentado com "frosting" (aspecto grisalho) bem evidente (A. lituratus tem ventre marrom claro, uniforme), a ausência do escudo rostral que caracteriza o crânio de $A$. lituratus. Além disso, as pernas e membrana interfemural são menos pilosos que em A. lituratus. Os exemplares do PEPB, em geral, não divergiram da descrição fornecida por HANDLEY (1989) quanto aos caracteres externos.

$\mathrm{O}$ antebraço de A. fimbriatus $(62,6-68,7 \mathrm{~mm})$ é mais curto que o de $A$. lituratus (68,5-74,2 mm), mas as dimensões craniais são comparáveis (HANDLEY 1989). Isso foi verdadeiro para os exemplares do PEPB, visto que as medidas craniais, com exceção da largura pós-orbital (Lpo), se sobrepuseram com as de $A$. lituratus. Não houve sobreposição em relação ao $\mathrm{AB}$, dentro da faixa acima citada para $A$. fimbriatus. A constrição pós-orbital (macho $=7,60$; fêmea $=7,54$ ) dos exemplares de $A$. fimbriatus é em média mais larga do que a dos exemplares de $A$. lituratus (macho $=7,28$; fêmea $=7,10$ ) estudados.

Em todos os exemplares, notou-se a ausência do terceiro molar superior. BARQUEZ \& OJEDA (1992) constataram a presença desse dente em exemplares de A. fimbriatus na Argentina.

Artibeus fimbriatus é uma espécie comum em florestas tropicais úmidas, próximo à latitude $25^{\circ} \mathrm{S}$, do nível do mar até cerca de aproximadamente $530 \mathrm{~m}$ (HANDley 1989; MARQues-Aguiar 1994). No PEPB, A. fimbriatus, com 139 indivíduos amostrados, foi a segunda espécie mais frequentemente capturada, superada apenas por A. lituratus com 265 indivíduos capturados.

\section{Artibeus lituratus (Olfers, 1818)}

Localidade tipo: Paraguai, Assunção.

Distribuição geográfica: Sinaloa e Tamaulipas (México) ao sul do Brasil, norte da Argentina, e Bolívia; Trinidad e Tobago; sul das Pequenas Antilhas; Ilhas Três Marias (KoOPMAN 1993; MARQues-Aguiar 1994).

Total de exemplares capturados: 265 .

Material examinado (16): macho ALP 5653; macho ALP 5656; macho ALP 5711; macho ALP 5736; macho ALP 5753; macho ALP 5829; fêmea ALP 5655; fêmea ALP 5659; fêmea ALP 5691; fêmea ALP 5705; fêmea ALP 5709; fêmea ALP 5771; fêmea ALP 5774; fêmea ALP 5789; fêmea ALP 5799; fêmea ALP 5803.

Os dados morfométricos referentes a Artibeus lituratus encontram-se na tabela VII.

Os exemplares amostrados pelo presente estudo foram identificados de acordo com os critérios morfométricos propostos por HANDLEY (1989), embora em medidas de Lz, Lm, Lcx e CM-S tenha ocorrido sobreposição com a variação reportada pelo autor para A. fimbriatus. As medidas de comprimento de antebraço e comprimento total do crânio dos exemplares coincidiram com as medidas fornecidas por KOEPCKE \& KRAFT (1984), HANDLEY (1989) e LiM \& WiLSON (1993) para A. lituratus.

\section{Artibeus obscurus Schinz, 1821}

Localidade tipo: Brasil, Bahia, Rio Peruhype, Vila Viçosa.

Distribuição geográfica: Colômbia, Venezuela, Guianas, Equador, Peru, Bolívia, Brasil (HANDLEY 1989; KOOPMAN 1993). 
Total de exemplares capturados: 20.

Material examinado (10): macho ALP 5682; macho ALP 5692; macho ALP 5696; macho ALP 5699; macho ALP 5763; macho ALP 5772; macho ALP 5812; macho ALP 5830; fêmea ALP 5675; fêmea ALP 5782.

Dados morfométricos referentes a essa espécie encontram-se na tabela VIII.

Tabela VIII. Medidas de exemplares de Artibeus obscurus do Parque Estadual da Pedra Branca, Rio de Janeiro. (N) Número de exemplares.

\begin{tabular}{|c|c|c|c|c|c|c|}
\hline \multirow{2}{*}{ Caracteres } & \multicolumn{3}{|c|}{ Machos } & \multicolumn{3}{|c|}{ Fêmeas } \\
\hline & Média & Mínimo-Máximo & $\mathrm{N}$ & Média & Minimo-Máximo & $\mathrm{N}$ \\
\hline$A B$ & 57,51 & $55,3-59,6$ & 7 & 59,20 & $59,1-59,3$ & 2 \\
\hline CT & 27,46 & $26,4-28,3$ & 7 & 28,10 & $27,9-28,3$ & 2 \\
\hline $\mathrm{Cb}$ & 24,84 & $24,1-25,7$ & 7 & 25,25 & $24,6-25,9$ & 2 \\
\hline $\mathrm{B}$ & 21,60 & $20,6-22,4$ & 7 & 22,55 & $22,1-23,0$ & 2 \\
\hline $\mathrm{Cpt}$ & 13,41 & $12,2-14.7$ & 7 & 14,15 & $13,9-14,4$ & 2 \\
\hline CM-S & 10,13 & $9,7-10,4$ & 7 & 10,45 & $10,4-10,5$ & 2 \\
\hline $\mathrm{CM}-\mathrm{I}$ & 11,12 & $10,8-11,3$ & 6 & 11,15 & $11,1-11,2$ & 2 \\
\hline $\mathrm{Lm}$ & 12,67 & $12,2-13,0$ & 6 & 12,65 & $12,5-12,8$ & 2 \\
\hline $\mathrm{Cm}$ & 17,95 & $17,3-18,4$ & 7 & 18,85 & $18,5-19,2$ & 2 \\
\hline LC & 7,87 & $7,4-8,1$ & 7 & 7,95 & $7,9-8,0$ & 2 \\
\hline Lpo & 6,51 & $6,2-6,7$ & 7 & 6,90 & $6,9-6,9$ & 2 \\
\hline $\mathrm{Lz}$ & 16,94 & $16,4-17,3$ & 7 & 17,20 & $17,0-17,4$ & 2 \\
\hline Lcx & 12,69 & $12,1-12,9$ & 7 & 12,80 & $12,8-12,8$ & 2 \\
\hline $\mathrm{Lmt}$ & 14,61 & $13,7-15,3$ & 7 & 14,50 & $14,0-15,0$ & 2 \\
\hline
\end{tabular}

A primeira referência a essa espécie foi feita por HANDLEY (1976) na Venezuela sob o nome Artibeus fuliginosus Gray, 1838.

Artibeus obscurus é uma espécie distinguida das outras de grandes Artibeus pelo tamanho relativamente pequeno, em relação às demais, pelagem longa e macia, coloração enegrecida, coloração ventral com "frosting" (aspecto grisalho), listras faciais obsoletas, membranas interfemurais nuas, presença do terceiro molar superior e rostro inflado anterodorsalmente, após os caninos, entre outros caracteres (HANDLEY 1989). Embora semelhante a Artibeus jamaicensis Leach, 1821, com a qual ocorre em simpatria, A. obscurus difere desta por caracteres externos e craniodentais e pelo tamanho, a primeira sendo um pouco maior e de coloração mais clara (HANDLEY 1989; MARQUES-AgUIAR 1994).

Os exemplares colecionados no PEPB foram identificados como A. obscurus, com base nos caracteres acima mencionados. A maioria das medidas coincidiu com as reportadas para A. obscurus por HANDLEY (1989), com pouca sobreposição com os valores mínimos das medidas referidas para A. jamaicensis pelo autor. Os caracteres morfológicos e medidas também correspondem à descrição de KOEPCKE \& KRAFT (1984), sob o nome A. fuliginosus, e MARQuES-AgUIAR (1994), para a espécie.

ANDERSON et al. (1982) mencionaram que a presença do terceiro molar superior, considerada importante para distinção das espécies de Artibeus (ANDERSEN 1908; TADDEI 1979; LIM \& WILSON 1993), é variável individualmente e HANDLEY (1989) ressalva que a presença desse dente está sujeita à variação 
geográfica, mencionando sua presença em $94 \%$ dos exemplares no norte da Amazônia (Amapá e Venezuela) e Colômbia, Equador e Peru e em 77\% dos exemplares do sul da Amazônia no Brasil.

No presente estudo, o terceiro molar superior estava presente, em ambos os lados, em apenas um dos exemplares e em um exemplar estava presente em apenas um lado; nos outros oito exemplares examinados, não se notou qualquer vestígio deste dente.

\section{Chiroderma doriae Thomas, 1891}

Localidade tipo: Brasil, Minas Gerais.

Distribuição geográfica: Sudeste do Brasil (KoOPMAN 1993). Todavia, GREGORIN (1998) confirmou a ocorrência dessa espécie para o Estado do Mato Grosso do Sul, o que amplia sua distribuição para o oeste do Brasil.

Total de exemplares capturados: 05 .

Material examinado (05): macho ALP 5761; macho ALP 5784; fêmea ALP 5684; fêmea ALP 5693; fêmea ALP 5714.

Dados morfométricos para $C$. doriae encontram-se na tabela IX. Estudos abordando a morfometria dessa espécie são raros, um deles foi publicado por TADDEI (1979), subsidiado por extensa coleção da região norte-ocidental do Estado de São Paulo. VIEIRA (1942) forneceu descrição para a espécie, porém nenhuma medida externa ou craniana.

Segundo TADDEI (1979), a descrição de $C$. doriae baseou-se em exemplar mal conservado e os caracteres externos descritos por Thomas e VIEIRA (1942) eram pouco consistentes. O autor complementou a descrição original com detalhes morfológicos externos e craniais e caracteres úteis para distinção entre $C$. doriae e Chiroderma villosum Peters, 1860, as quais ocorrem simpatricamente na região Sudeste do Brasil (KoOPMAN 1994).

Entre caracteres diagnósticos para $C$. doriae, TADDEI (1979) destacou o comprimento do antebraço (49,5-55,5 mm), listras faciais claras e evidentes e listra dorsal branca mais distinta que em C. villosum (antebraço de 44,5 a 50,0 mm), extendendo-se da base da cabeça ao extremo posterior do corpo, até a base do uropatágio, os mesmos já mencionados por VIZOTTO \& TADDEI (1973).

Em um exemplar estudado a listra dorsal mostrou-se apenas esboçada, mas todos apresentaram as quatro listras faciais perfeitamente distintas e comprimento de antebraço dentro da variação acima referida para $C$. doriae, conforme pode ser observado na tabela IX. Cabe ressaltar que todos os exemplares apresentaram o primeiro pré-molar inferior com cúspide anterior distinta, caráter listado por TADDEI (1979) e Koopman (1994) como importante para distinguir $C$. doriae de $C$. villosum.

\section{Platyrrhinus lineatus (E. Geoffroy, 1810)}

Localidade tipo: Paraguai, Assunção.

Distribuição geográfica: Colômbia ao Peru, Bolívia, Uruguai, norte da Argentina e sul e leste do Brasil; Guiana Francesa e Suriname (KOOPMAN 1993).

Total de exemplares capturados: 05 . 
Material examinado (04): macho ALP 5690; fêmea ALP 5703; fêmea ALP 5827; fêmea ALP 5858.

Os dados morfométricos obtidos são apresentados na tabela IX.

Tabela IX. Medidas de exemplares de Chiroderma doriae e Platyrrhinus lineatus do Parque Estadual da Pedra Branca, Rio de Janeiro. (N) Número de exemplares.

\begin{tabular}{|c|c|c|c|c|c|c|c|c|c|c|}
\hline \multirow{3}{*}{ Caracteres } & \multicolumn{6}{|c|}{ Chiroderma doriae } & \multicolumn{4}{|c|}{ Platyrrhinus lineatus } \\
\hline & \multicolumn{3}{|c|}{ Machos } & \multicolumn{3}{|c|}{ Fêmeas } & \multirow[t]{2}{*}{ Machos } & \multicolumn{3}{|c|}{ Fêmeas } \\
\hline & Média & Mín-Máx & $\mathrm{N}$ & Média & Mín-Máx & $\mathrm{N}$ & & Média & Mín-Máx & N \\
\hline$A B$ & 53,40 & $53,1-53,7$ & 2 & 51,30 & $50,6-52,0$ & 3 & 46,2 & 46,80 & $45,5-58,1$ & 3 \\
\hline CT & 29,10 & $28,8-29,4$ & 2 & 28,73 & $28,4-29,1$ & 3 & 25,2 & 25,45 & $25,2-25,6$ & 3 \\
\hline $\mathrm{Cb}$ & 27,15 & $27,0-27,3$ & 2 & 26,97 & $26,8-27,1$ & 3 & 23,0 & 22,95 & $22,3-23,3$ & 3 \\
\hline B & 24,75 & $24,7-24,8$ & 2 & 24,30 & $24,2-24,5$ & 3 & 20,3 & 20,40 & $20,0-20,6$ & 3 \\
\hline Cpt & 15,90 & $15,7-16,1$ & 2 & 15,57 & $15,3-15,7$ & 3 & 12,5 & 12,13 & $11,7-12,4$ & 3 \\
\hline $\mathrm{CM}-\mathrm{S}$ & 10,75 & $10,7-10,8$ & 2 & 10,60 & $10,3-10,9$ & 3 & 9.0 & 9,20 & $9,1-9,3$ & 3 \\
\hline $\mathrm{CM}-\mathrm{I}$ & 11,55 & $11,5-11,6$ & 2 & 11,33 & $11.1-11,5$ & 3 & 9,9 & 9,85 & $9,7-10,0$ & 3 \\
\hline Lm & 13,00 & $12,8-13,2$ & 2 & 12,77 & $12,3-13,1$ & 3 & 10,4 & 10,45 & $10,3-10,5$ & 3 \\
\hline $\mathrm{Cm}$ & 20,40 & $20,3-20,5$ & 2 & 20,37 & $20,2-20,6$ & 3 & 16,5 & 16,60 & $16,5-16,9$ & 3 \\
\hline LC & 6,35 & $6,3-6,4$ & 2 & 6,43 & $6,2-6,6$ & 3 & 6,4 & 6,48 & $6,2-6,7$ & 3 \\
\hline Lpo & 6,60 & $6,6-6,6$ & 2 & 6,20 & $6,0-6,5$ & 3 & 6,5 & 6,18 & $6,0-6,4$ & 3 \\
\hline $\mathrm{Lz}$ & 18,25 & $17,8-18,7$ & 2 & 17,57 & $17,2-18,0$ & 3 & 14,5 & 14,20 & $13,9-14,8$ & 3 \\
\hline LCx & 12,15 & $12,0-12,3$ & 2 & 11,93 & $11,5-12,2$ & 3 & 10,9 & 10,50 & $10,4-10,8$ & 3 \\
\hline $\mathrm{Lmt}$ & 13,80 & $13,8-13,8$ & 2 & 13,50 & $13,4-13,6$ & 3 & 12,4 & 12,05 & $12,0-12,1$ & 3 \\
\hline
\end{tabular}

Segundo TADDEI (1979), P. lineatus (AB = 43,7 a 50,1 mm) e Platyrrhinus recifinus (Thomas, 1901)( $\mathrm{AB}=41,0$ a 43,0 $\mathrm{mm})(c f$. SANBORN 1955) apresentam dimensões intermediárias entre Platyrrhinus infuscus (Peters, 1880) $(\mathrm{AB}=55,7 \mathrm{a}$ $59,1 \mathrm{~mm}$ ) (cf. GARDNER \& CARTER 1972) e as menores Platyrrhinus brachycephallus (Rouk \& Carter, 1972) ( $\mathrm{AB}=36,4$ a 42,0 mm) e Platyrrhinus helleri (Peters, $1866)(\mathrm{AB}=35,5 \mathrm{a} 41,3 \mathrm{~mm})(c f$. ROUK \& CARTER 1972).

Um macho e três fêmeas de Platyrrhinus apresentaram medidas dentro da variação proposta por SANBORN (1955), SWANEPOEL \& GENOWAYS (1979) e também dentro dos extremos de AB e Cb mencionados por KoOPMAN (1994) para $P$. lineatus.

Além disso, os quatro exemplares citados apresentaram incisivos superiores centrais grandes, robustos e em contato, além de incisivos inferiores distintamente bilobulados, condição encontrada em $P$. lineatus, conforme CARTER \& DOLAN (1978) e WiLlig \& HOLLANDER (1987). Outras diferenças foram encontradas em relação aos dois exemplares de $P$. recifinus, no que diz respeito à série de dentes superiores (ver discussão para $P$. recifinus).

\section{Platyrrhinus recifinus (Thomas, 1901)}

Localidade tipo: Brasil, Pernambuco, Recife.

Distribuição geográfica: Leste do Brasil (KoOPMAN 1993).

Total de exemplares capturados: 02 .

Material examinado (02): macho ALP 5662; macho ALP 5828. 
As medidas obtidas para essa espécie encontram-se na tabela X. Não há informações detalhadas na literatura a respeito da morfometria desta espécie, cuja posição taxonômica ainda é objeto de discussão entre os sistematas.

Thomas (1901) descreveu Platyrrhinus recifinus a partir de um único exemplar, macho, procedente de Pernambuco, sustentando que esta forma poderia ser distinguida de $P$. lineatus por seus incisivos superiores de tamanho diminuto e separados um do outro. SANBORN (1955) mencionou que o tamanho e a disposição dos incisivos não constituem caracteres constantes e seguros para separar $P$. recifinus de $P$. lineatus. Esse autor relatou que $P$. recifinus cujo comprimento de antebraço varia de 41,0 a 43,0 mm, é menor que $P$. lineatus e maior que $P$. helleri (vide discussão para $P$. lineatus).

Segundo Willig \& Hollander (1987), quatro medidas craniais de $P$. recifinus sobrepõem-se com as de $P$. lineatus. Nesse sentido, $P$. recifinus é uma espécie questionada por alguns autores (ROUK \& CARTER 1972; JONES \& CARTER 1976). Não obstante, grande parte dos autores (e.g. VIEIRA 1955; CABRERA 1958; VizotTo \& TADDEI 1973; SWANEPOEL \& GENOWAYS 1979; OWEN 1988; KoOPMAN 1982; 1993; 1994; PERACCHI \& ALBUQUERQUE 1993) têm seguido SANBORN (1955), tratando $P$. recifinus como espécies válida. Análises fenéticas e multivariadas conduzidas por OWEN (1988) revelaram que $P$. recifinus é mais estreitamente relacionada com $P$. helleri do que com $P$. lineatus.

Dois indivíduos de Platyrrhinus apresentaram a maioria das medidas, inclusive $\mathrm{AB}$ dentro dos extremos fornecidos por SANBORN (1955) e SWANEPOEL \& GENOWAYS (1979) para $P$. recifinus, tendo ocorrido sobreposição com os valores mínimos de $P$. lineatus em Lz, Lcx, CM-S e Lm, fornecidos pelos últimos autores.

Os exemplares apresentaram antebraço pouco maior que a medida do holótipo, examinado por CARTER \& DOLAN (1978), conforme mostra a tabela VIII. As médias das dimensões cranianas obtidas em geral coincidem com as do holótipo (Tab. X).

Tabela X. Medidas obtidas de dois machos de Platyrrhinus recifinus do Parque Estadual da Pedra Branca, Rio de Janeiro, associadas a medidas do holótipo, tomadas por CARTER \& DOLAN (1978).

\begin{tabular}{lcrrr}
\hline Caracteres & ALP 5662 & ALP 5828 & Médias & Holótipo' $^{{ }^{\prime}}$ \\
\hline AB & 41,70 & 9,5 & 41,85 & $40,2^{*}$ \\
CT & 23,90 & 16,0 & 24,05 & 24,1 \\
Cb & 21,60 & 6,0 & 21,80 & 21,7 \\
Lz & 13,80 & 10,2 & 13,95 & 14,0 \\
Lmt & 12,30 & 8,8 & 12,00 & 11,7 \\
Lcx & 10,30 & 5,7 & 10,40 & 10,3 \\
Lpo & 5,50 & 10,5 & 5,60 & 5,6 \\
CM-S & 8,70 & 11,7 & 8,75 & 8,9 \\
Lm & 10,20 & 14,1 & 10,30 & 10,5 \\
LC & 6,10 & 22,0 & 6,05 & 6,4 \\
Cm & 15,60 & 24,2 & 15,80 & 15,3 \\
CM-1 & 9,50 & 42,0 & 9,50 & 9,5 \\
\hline
\end{tabular}

(1) B.M. 81,$3 ; 16,4 ;\left(^{*}\right) \mathrm{AB}=41,0 \mathrm{~mm}$ (medido por THOMAs 1901). 
Apesar da advertência de SANBORN (1955), o tamanho e disposição dos incisivos centrais superiores foram mantidos por VIzOTTO \& TADDEI (1973) e KOOPMAN (1994), como critérios para separação das espécies. Ambos os exemplares apresentavam os incisivos superiores menores (não robustos) e ligeiramente separados, condição não observada nos quatro exemplares identificados como $P$. lineatus.

Encontrou-se diferenças também em relação às series de dentes superiores e inferiores: os dois exemplares apresentaram cúspides anteriores dos primeiros molares inferior e superior distintamente aguçadas, desenvolvidas, além do primeiro e segundo pré-molares inferiores estreitos e bem aguçados, condições não encontradas para os exemplares referidos a $P$. lineatus. Nesses, pré-molares e molares são baixos, com cúspides baixas e não aguçadas.

As condições observadas no material atribuído a $P$. recifinus também foram observadas num exemplar dessa espécie, procedente de Pernambuco (localidade Serra dos Cavalos), por empréstimo a A. L. Peracchi, para estudo.

\section{Sturnira Iilium (E. Geoffroy, 1810)}

Localidade tipo: Paraguai, Assunção.

Distribuição geográfica: Pequenas Antilhas; Sonora e Tamaulipas (México) ao norte da Argentina, Uruguai e leste do Brasil; Trinidad e Tobago; provavelmente Jamaica (KOOPMAN 1993).

Total de exemplares capturados: 27.

Material examinado (14): macho ALP 5694; macho ALP 5718; macho ALP 5735; macho ALP 5738; macho ALP 5810; fêmea ALP 5689; fêmea ALP 5697; fêmea ALP 5719; fêmea ALP 5720; fêmea ALP 5739; fêmea ALP 5762; fêmea ALP 5773; fêmea ALP 5809; fêmea ALP 5811.

Dados morfométricos referentes a Sturnira lilium encontram-se na tabela XI.

PACHECO \& PATTERSON (1991) confirmaram a colocação do gênero Sturnira Gray, 1842 dentro da subfamília Stenodermatinae. OwEN (1988) citou o gênero Sturnira como um grupo feneticamente atípico e bem definido dessa subfamília.

KOOPMAN $(1993,1994)$ citou duas espécies do gênero com ocorrência confirmada em território brasileiro, S. lilium e Sturnira tildae de la Torre, 1959, as quais ocorrem simpatricamente no Sudeste do Brasil e um registro duvidoso de Sturnira bidens Thomas, 1915 para a Amazônia brasileira. Uma chave para identificação das espécies de Sturnira foi publicada por DAVIS (1980).

A condição serrada do primeiro e do segundo molares inferiores, devido à separação das cúspides linguais por entalhes profundos e bem definidos e a curvatura para fora dos arcos zigomáticos, apontados por DAVIS (1980), GANNON et al. (1989) e KoOPMAN (1994), como caracteres úteis para separar S. lilium de S. tildae, foram observados em todos os exemplares estudados. SimmONS \& Voss (1998) relataram ter identificado satisfatoriamente exemplares de S. lilium com base na morfologia das cúspides linguais dos molares inferiores. 


\section{Vampyressa pusilla (Wagner, 1843)}

Localidade tipo: Brasil, Rio de janeiro, Sapitiba.

Distribuição geográfica: Oaxaca e Veracruz (México) a Bolívia e Guianas; Paraguai e sudeste do Brasil (KoOPMAN 1993). A espécie foi confirmada para a Argentina por MARES et al. (1995).

Total de exemplares capturados: 07.

Material examinado (05): macho ALP 571; macho ALP 5717; macho ALP 5731; macho ALP 5783; macho ALP 5831.

Dados morfométricos obtidos para três machos adultos encontram-se na tabela XI. Estudos abordando dados morfométricos sobre $V$. pusilla são escassos.

Tabela XI. Medidas de exemplares de Sturnira lilium e Vampyressa pusilla do Parque Estadual da Pedra Branca, Rio de Janeiro. (N) Número de exemplares.

\begin{tabular}{|c|c|c|c|c|c|c|c|c|c|}
\hline \multirow{3}{*}{ Caracteres } & \multicolumn{6}{|c|}{ Sturnira lilium } & \multicolumn{3}{|c|}{ Vampyressa pusilla } \\
\hline & \multicolumn{3}{|c|}{ Machos } & \multicolumn{3}{|c|}{ Fêmeas } & \multicolumn{3}{|c|}{ Machos } \\
\hline & Média & Mín-Máx & $\mathrm{N}$ & Média & Mín-Máx & $\mathrm{N}$ & Média & Mín-Máx & $\mathrm{N}$ \\
\hline$A B$ & 44,20 & $43,0-44,9$ & 5 & 42,66 & $39,4-44,1$ & 9 & 33,90 & $33,5-34,4$ & 3 \\
\hline CT & 23,54 & $22,8-24,1$ & 5 & 22,89 & $22,3-23,5$ & 9 & 20,10 & $20,0-20,3$ & 3 \\
\hline $\mathrm{Cb}$ & 21,40 & $20,2-21,7$ & 5 & 20,58 & $19,9-21,0$ & 9 & 18,20 & $18,1-18,4$ & 3 \\
\hline B & 18,84 & $18,2-19,5$ & 5 & 18,10 & $17,5-18,7$ & 9 & 16,17 & $16,0-16,3$ & 3 \\
\hline Cpt & 10,24 & $9,8-10,9$ & 5 & 9,80 & $9,3-10,3$ & 9 & 9,33 & $9,2-9,5$ & 3 \\
\hline CM-S & 6,88 & $6,7-7,2$ & 5 & 6,68 & $6,5-6,8$ & 9 & 6,70 & $6,5-6,9$ & 3 \\
\hline $\mathrm{CM}-\mathrm{I}$ & 7,70 & $7,5-7,9$ & 5 & 7,46 & $7,1-7,8$ & 9 & 7,00 & $6,8-7,2$ & 3 \\
\hline $\mathrm{Lm}$ & 8,38 & $8,2-8,7$ & 5 & 8,29 & $8,0-8,6$ & 9 & 8,10 & $7,9-8,2$ & 3 \\
\hline $\mathrm{Cm}$ & 15,16 & $14,9-15,7$ & 5 & 14,63 & $14,3-15,0$ & 9 & 12,77 & $2,6-12,9$ & 3 \\
\hline LC & 6,50 & $6,3-6,9$ & 5 & 6,20 & $6,0-6,7$ & 9 & 4,87 & $4,7-5,0$ & 3 \\
\hline Lpo & 6,08 & $6,0-6,3$ & 5 & 6,10 & $5,7-6,4$ & 9 & 5,27 & $5,2-5,4$ & 3 \\
\hline $\mathrm{Lz}$ & 14,20 & $14,0-14,5$ & 5 & 13,74 & $13,2-14,4$ & 9 & 11,53 & $11,4-11,7$ & 3 \\
\hline LCx & 10,66 & $10,4-10,8$ & 5 & 10,50 & $10,2-10,8$ & 9 & 9.07 & $8,7-9,3$ & 3 \\
\hline Lmt & 12,70 & $12,3-13,3$ & 5 & 12,32 & $11,5-12,7$ & 9 & 9,90 & $9,7-10,0$ & 3 \\
\hline
\end{tabular}

Outras duas espécies de Vampyressa Thomas, 1900 têm ocorrência confirmada no Brasil: Vampyressa bidens (Dobson, 1878) e Vampyressa brocki Peterson, 1968, mas somente na Amazônia brasileira (KoOPMAN 1993).

Vampyressa nattereri, cujo tipo é um macho adulto, foi descrita por GoODWIN (1963), mas não teve validade reconhecida por PETERSON (1968), que advertiu que as diferenças encontradas por GooDWIN (1963) devem-se ao fato do tipo de Phyllostoma pusillum (Wagner, 1843) ser um macho subadulto. Assim, V. nattereri passou a ser considerada sinônimo de $V$. pusilla, arranjo aceito por autores subsequentes (JONES \& CARTER 1976; LEWIS \& WILSON 1987; KOOPMAN 1982; 1993).

\section{Desmodontinae Bonaparte, 1845}

\section{Desmodus rotundus (E. Geoffroy, 1810)}

Localidade tipo: Paraguai, Assunção.

Distribuição geográfica: Uruguai, norte da Argentina, e norte do Chile até 
Sonora, Nuevo Leon e Tamaulipas (México); Ilha Margarita (Venezuela); Trinidad (KOOPMAN 1993).

Total de exemplares capturados: 41 .

Material examinado (02): macho ALP 5681; macho ALP 5752.

Medidas obtidas de machos de Desmodus rotundus são apresentados na tabela XII.

Tabela XII. Medidas de machos de Desmodus rotundus e de Diphylla ecaudata do Parque Estadual da Pedra Branca, Rio de Janeiro. (N) Número de exemplares.

\begin{tabular}{|c|c|c|c|c|c|c|c|c|}
\hline \multirow{3}{*}{$\begin{array}{l}\text { Caracteres } \\
A B\end{array}$} & \multicolumn{4}{|c|}{ Desmodus rotundus } & \multicolumn{4}{|c|}{ Diphylla ecaudata } \\
\hline & \multirow{2}{*}{$\begin{array}{r}\text { Média } \\
56,50\end{array}$} & \multicolumn{2}{|c|}{ Minimo-Máximo } & \multirow{2}{*}{$\frac{N}{2}$} & \multirow{2}{*}{$\begin{array}{r}\text { Média } \\
53,80\end{array}$} & \multicolumn{2}{|c|}{ Mínimo-Máximo } & \multirow{2}{*}{$\frac{N}{4}$} \\
\hline & & 54,0 & 59,0 & & & 53,0 & 54,7 & \\
\hline CT & 25,10 & 25,1 & 25,1 & 2 & 23,70 & 23,3 & 24,0 & 4 \\
\hline $\mathrm{Cb}$ & 22,25 & 22,1 & 22,4 & 2 & 21,03 & 20,5 & 21,6 & 4 \\
\hline B & 19,55 & 19,5 & 19,6 & 2 & 18,00 & 17,2 & 18,6 & 4 \\
\hline $\mathrm{Cpt}$ & 9.40 & 9,3 & 9.5 & 2 & 7,28 & 7,0 & 7,5 & 4 \\
\hline CM-S & 3,70 & 3,7 & 3,7 & 2 & 3,48 & 3,3 & 3,8 & 4 \\
\hline $\mathrm{CM}-1$ & 5,30 & 5.0 & 5,6 & 2 & 3,88 & 3,4 & 4,1 & 4 \\
\hline Lm & 6,30 & 6,3 & 6,3 & 2 & 6,13 & 5,9 & 6,4 & 4 \\
\hline $\mathrm{Cm}$ & 15,10 & 15,0 & 15,2 & 2 & 13,70 & 13,0 & 14,3 & 4 \\
\hline Lc & 6,60 & 6,6 & 6,6 & 2 & 5,90 & 5,8 & 6,1 & 4 \\
\hline Lpo & 5,60 & 5,5 & 5,7 & 2 & 7,00 & 6,6 & 7,4 & 4 \\
\hline Lz & 12,10 & 12,0 & 12,2 & 2 & 13,00 & 12,6 & 13,5 & 4 \\
\hline LCX & 12,00 & 11,7 & 12,3 & 2 & 11,93 & 11,7 & 12,1 & 4 \\
\hline Lmt & 12,30 & 11,9 & 12,7 & 2 & 12,35 & 12,0 & 12,9 & 4 \\
\hline
\end{tabular}

Numerosos autores têm apresentado dados morfométricos para $D$. rotundus, entre os quais GoOdWIN \& GREENHALl (1961), Husson (1962), SWANEPOEL \& GENOWAYS (1979), GREENHALl et al. (1983), MARES et al. (1996) e SimMONS \& Voss (1998). Para o Brasil, dados morfométricos para $D$. rotundus podem ser encontrados no estudo de WILLIG (1983), sobre extensa coleção procedente do Nordeste brasileiro.

\section{Diphylla ecaudata Von Spix, 1823}

Localidade tipo: Brasil, Bahia, Rio São Francisco.

Distribuição geográfica: Sul do Texas (Estados Unidos) a Venezuela, Peru, Bolívia e leste do Brasil (KoOpman 1993).

Total de exemplares capturados: 04 .

Material examinado (04): macho ALP 5746; macho ALP 5851; macho ALP 5852; macho ALP 5853.

Medidas para exemplares de Diphylla ecaudata encontram-se na tabela XII. Dados morfométricos para a espécie foram sumarizados por SWANEPOEL \& GENOWAYS (1979) e GREENHALL et al. (1984). 


\section{Vespertilionidae Gray, 1821}

\section{Eptesicus brasiliensis (Desmarest, 1819)}

Localidade tipo: Brasil, Goiás.

Distribuição geográfica: Veracruz (México) até o norte da Argentina e Uruguai; Trinidad e Tobago (KoOPMAN 1993).

Total de exemplares capturados: 01 .

Material examinado (01): fêmea ALP 5825.

Medidas obtidas de uma fêmea dessa espécie são apresentadas na tabela XIII.

Em revisão de morcegos sul-americanos do gênero Eptesicus Rafinesque, 1820 DAVIS (1966) propôs os seguintes caracteres para identificação de E. brasiliensis: comprimento da série de dentes superiores maior que $6 \mathrm{~mm}$ (5,7-6,7 mm), metacarpo III medindo $37 \mathrm{~mm}$ ou mais $(35,8-42,5 \mathrm{~mm})$, comprimento do antebraço normalmente 40 mm ou mais (39,6-45,6 mm) e comprimento do crânio entre 16 e 18 mm.

Segundo WiLliams (1978), há um gradiente crescente de tamanho entre Eptesicus diminutus Osgood, 1915, E. brasiliensis, e Eptesicus furinalis (d'Orbigny, 1847), com sobreposição entre pares de espécies. Ainda segundo o autor, o comprimento da mandíbula e da série de dentes inferiores são bons caracteres para distinguir E. brasiliensis $(\mathrm{Cm}=12,6$ a $12,9 \mathrm{~mm}$ e $\mathrm{CM}-\mathrm{I}=6,4$ a 7,2 $\mathrm{mm})$. Todos os caracteres citados mostraram satisfatórios para identificar o exemplar em estudo.

Os trabalhos disponíveis a respeito da morfometria de E. brasiliensis são escassos e caracterizados por amostragens muito reduzidas tomadas de localidades isoladas, ou até mesmo por um único exemplar (e.g. VIEIRA 1942).

\section{Myotis nigricans (Schinz, 1821)}

Localidade tipo: Brasil, Espírito Santo, entre os rios Itapemirim e Iconha.

Distribuição geográfica: Nayarit e Tamaulipas (México) ao Peru, norte da Argentina, e sul do Brasil; Trinidad e Tobago; Granada (Pequenas Antilhas) (KOOPMAN 1993).

Total de exemplares capturados: 11 .

Material examinado (05): macho ALP 5742; macho ALP 5744; macho ALP 5861; fêmea ALP 5727; fêmea ALP 5821.

Os dados morfométricos para Myotis nigricans são apresentados na tabela XIII.

Segundo a revisão de LAVAL (1973), M. nigricans é uma espécie altamente variável, normalmente sem crista sagital ou se presente, esta é pouco desenvolvida. Além disso, em algumas regiões ao longo de sua distribuição, $M$. nigricans ocorre simpatricamente com várias espécies conhecidas da região Neotropical e certos indivíduos podem se assemelhar a exemplares de outras espécies, o que dificulta a identificação correta.

BARQUEZ \& OJEDA (1992), por exemplo, mencionaram que os exemplares de M. nigricans eram facilmente confundidos com Myotis riparius Handley, 1960, na região do Chaco Argentino. Nas regiões de Caatinga e Cerrado, no Nordeste brasileiro, uma coleção de Myotis, a princípio atribuída a M. nigricans (MARES et al. 1981; WILLIG 1983), na realidade tratava-se de $M$. riparius (WILLIG \& MARES 1989). 
Indivíduos de Myotis albescens (E. Geoffroy, 1806) que não exibem a distinta aparência fosca ou grisalha da pelagem também estão sujeitos a confusão com M. nigricans, dos quais se distinguem pela morfologia cranial; por outro lado, alguns representantes dessas duas espécies possuem crânio muito semelhante, distinguindo-se, então, pela coloração da pelagem (LAVAL 1973). M. riparius e $M$. albescens são espécies com ocorrência confirmada na Mata Atlântica do Sul e Sudeste do Brasil ( $c f$. MARINHO-FILHO 1996).

Em todos os exemplares, notou-se a ausência de crista sagital, pelagem uniformemente colorida, ausência de franja de pêlos na margem posterior do uropatágio, proporção Lc/Lpo menor que 1, pré-molares superiores em fileira reta, sem deslocamento do segundo pré-molar, estando portanto de acordo com os caracteres descritivos propostos por LAVAL (1973) para M. nigricans.

\section{Molossidae Gervais, 1856}

\section{Molossus molossus (Pallas, 1766)}

Localidade tipo: Martinica (Pequenas Antilhas).

Distribuição geográfica: Sinaloa e Coahuila (México) ao Peru, norte da Argentina, Uruguai, Brasil e Guianas; Grandes e Pequenas Antilhas; Ilha Margarita (Venezuela); Curaçao e Bonaire; Trinidad e Tobago (KoOPMAN 1993).

Total de exemplares capturados: 08.

Material examinado (03): macho ALP 5658; fêmea ALP 5724; fêmea ALP 5725.

Os dados morfométricos para Molossus molossus se encontram na tabela XIII. Para o Brasil, com exceção do estudo de WILLIG (1983), as informações a respeito da morfometria da espécie são baseadas em poucos exemplares.

Tabela XIII. Medidas de exemplares de Eptesicus brasiliensis, Myotis nigricans e Molossus molossus do Parque Estadual da Pedra Branca, Rio de Janeiro. (N) Número de exemplares.

\begin{tabular}{|c|c|c|c|c|c|c|c|c|c|c|c|}
\hline \multirow{3}{*}{ Caracteres } & \multirow{3}{*}{$\frac{\text { Eptesicus brasiliensis }}{\text { Fêmeas }}$} & \multicolumn{6}{|c|}{ Myotis nigricans } & \multicolumn{4}{|c|}{ Molossus molossus } \\
\hline & & \multicolumn{3}{|c|}{ Machos } & \multicolumn{3}{|c|}{ Fêmeas } & \multirow[t]{2}{*}{ Machos } & \multicolumn{3}{|c|}{ Fêmeas } \\
\hline & & Média & Mín-Máx & $\mathrm{N}$ & Média & Mín-Máx & $\mathrm{N}$ & & Média & Min-Máx & N \\
\hline$A B$ & 44,8 & 32,93 & $32,6-33,4$ & 3 & 33,30 & $33,1-33,5$ & 2 & 39,0 & 37,50 & $37,4-37,6$ & 2 \\
\hline CT & 17,6 & 13,40 & $13,2-13,5$ & 3 & 13,50 & $13,4-13,6$ & 2 & 18,4 & 17,10 & $17,0-17,2$ & 2 \\
\hline $\mathrm{Cb}$ & 16,5 & 12,67 & $12,4-12,8$ & 3 & 12,45 & $12,0-12,9$ & 2 & 16,4 & 15,20 & $15,1-15,3$ & 2 \\
\hline B & 14,9 & 10,17 & $10,0-10,3$ & 3 & 10,15 & $10,1-10,2$ & 2 & 14,3 & 13,10 & $13,0-13,2$ & 2 \\
\hline $\mathrm{Cpt}$ & 6,4 & 5,63 & $5,3-6,1$ & 3 & 5,25 & $5,0-5,5$ & 2 & 6,6 & 6,15 & $6,1-6,2$ & 2 \\
\hline $\mathrm{CM}-\mathrm{S}$ & 6,5 & 5,10 & $5,0-5,2$ & 3 & 5.05 & $5,0-5,1$ & 2 & 6,4 & 6,05 & $6,0-6,1$ & 2 \\
\hline $\mathrm{CM}-\mathrm{I}$ & 7.0 & 5,30 & $5,2-5,4$ & 2 & 5,35 & $5,3-5,4$ & 2 & 7,2 & 6,75 & $6.7-6.8$ & 2 \\
\hline $\mathrm{Lm}$ & 7,2 & 5,13 & $5,0-5,3$ & 3 & 5,05 & $5,0-5,1$ & 2 & 7,9 & 7,60 & $7,5-7,7$ & 2 \\
\hline $\mathrm{Cm}$ & 12,7 & 9,50 & $9,3-9,7$ & 2 & 9,55 & $9,4-9,7$ & 2 & 12,3 & 11,40 & $11,4-11,4$ & 2 \\
\hline Lc & 5,5 & 3,33 & $3,3-3,4$ & 3 & 3,40 & $3,4-3,4$ & 2 & 4,8 & 4,45 & $4,4-4,5$ & 2 \\
\hline Lpo & 4,1 & 3,63 & $3,6-3,7$ & 3 & 3,45 & $3,3-3,6$ & 2 & 4,1 & 3,80 & $3,8-3,8$ & 2 \\
\hline $\mathrm{Lz}$ & 11,7 & 7,80 & $7,4-8,3$ & 3 & 7,50 & 7,5 & 1 & 11,8 & 11,15 & $11,1-11,2$ & 2 \\
\hline Lcx & 8,0 & 6,43 & $6,3-6,6$ & 3 & 6.40 & $6,4-6,4$ & 2 & 9,4 & 9,00 & $8,9-9,1$ & 2 \\
\hline $\mathrm{Lmt}$ & 8,9 & 6.90 & $6,8-7,0$ & 3 & 6,85 & $6,8-6,9$ & 2 & 11,2 & 10,50 & $10,5-10,5$ & 2 \\
\hline
\end{tabular}


Dolan (1989) verificou que os exemplares do Peru e Equador possuem coloração mais escura, tendendo ao negro; os do Suriname tendendo ao marrom e os da Venezuela e Argentina mais claros, com coloração próxima ao castanho e argumentou que essa variabilidade pode estar ligada a áreas geográficas distintas, servindo de base para distinguir subespécies ou simplesmente refletir influência sazonal. Os exemplares analisados por MYERS \& WETZEL (1983) da região do Chaco Paraguaio apresentavam coloração mais clara que as populações de áreas mais mésicas, o que segundo os autores poderia estar relacionado ao clima semiárido da região. Os exemplares do PEPB, localizado numa região de clima úmido, apresentaram coloração castanho escura, brilhante, aproximando-se do negro.

\begin{abstract}
AGRADECIMENTOS. Somos gratos aos biólogos Vítor de Souza Ferreira, Alexandre Pinhão da Cruz, Gustavo Amaral Borges, Fabrício Olímpio de Lima e André Mariz da Silva, pelo auxílio nos trabalhos de campo e de laboratório; à Administração do Parque Estadual da Pedra Branca (Fundação Instituto Estadual de Florestas/Rio de Janeiro), pela autorização e oportunidade de realizar trabalhos de campo na área do Parque e, ainda, aos servidores do Instituto de Biologia da Universidade Federal Rural do Rio de Janeiro, pelo auxílio nos momentos necessários.
\end{abstract}

\title{
REFERÊNCIAS BIBLIOGRÁFICAS
}

Alonso-MejíA, A. \& R.A. Medellín. 1991. Micronycteris megalotis. Mamm. Species 376: 1-6.

Alvarez, J.; M. R. Willig; J.K. Jones Jr. \& W.D. Webster. 1991. Glossophaga soricina. Mamm. Species 379: 1-7.

ANDERSEN, K. 1908. A monograph of the chiropteran genera Uroderma, Enchistenes, and Artibeus. Proc.

Zool. Soc. London 1908: 204-319.

Anderson, S., K.F. Koopman \& G.K. Creighton. 1982. Bats of Bolívia: an annotated checklist. Amer.

Mus. Novit. 2750: 1-24.

Ávila-Pires, F.D. \& E. GouvÊA. 1977. Mamíferos do Parque Nacional de Itatiaia. Bol. Mus. Nac. Rio de Janeiro, n. sér. Zool. 291: 1-29.

Baptista, M. \& J.A. Oliveira. 1998. New records of Lonchophylla bokermanni (Chiroptera, Lonchophyllinae). Abstracts $11^{\text {th }}$ International Bat Research Conference, p. 47.

Barquez, R.M. \& R.A. OJedA. 1992. The bats (Mammalia: Chiroptera) of the Argentine Chaco. Ann. Carnegie Mus. 61 (3): 239-261.

Bernard, E. 2001. First capture of Micronycteris homezi Pirlot (Chiroptera, Phyllostomidae) in Brazil. Revta bras. Zool. 18 (2): 645-647.

Cabrera, A. 1958. Catálogo de los mamiferos de América del Sur. Rev. Mus. Argent. Cienc. Nat. "Bernardino Rivadavia", Cien. Zool., 4 (1): XVI+307.

CARTER, D.C. \& P.G. Dolan. 1978. Catalogue of type specimens of Neotropical bats in selected European Museums. Spec. Publ. Mus., Texas Tech Univ. 15: 1-136.

Davis, W.B. 1966. Review of South American bats of the genus Eptesicus. The Southwestern Naturalist 11 (2): 245-274.

1980. New Sturnira (Chiroptera: Phyllostomidae) from Central and South America, with key to currently recognized species. Occas. Pap. Mus., Texas Tech Univ. 70: 1-5.

DAVIS, W.B. \& D.C. CARTER. 1978. A review of the round-eared bats of the Tonatia silvicola complex, with descriptions of three new taxa. Occas. Pap. Mus., Texas Tech Univ. 53: 1-12.

Dolan, P. G. 1989. Systematics of Middle American mastiff bats of the genus Molossus. Spec. Publ.

Mus., Texas Tech Univ. 29: 1-71.

EsBERÁRD, C.E.L. 1998. Validade dos parâmetros da IUCN em amostra regional - há espécies de

Revta bras. Zool. 19 (Supl. 2): $113-140,2002$ 
morcegos ameaçados de extinção no Município do Rio de Janeiro?. Bol. Fund. Bras. Cons. Nat., Rio de Janeiro, 24: 71-86.

Fernandes, A. \& P. Bezerra. 1990. Estudo Fitogeográfico do Brasil. Fortaleza, Stylus Comunicações, $205 p$.

Gannon, M.R.; M.R. Willig \& J.K. Jones Jr. 1989. Sturnira lilium. Mamm. Species 333: 1-5.

Gardner, A.L. \& D.C. CARTer. 1972. Review of the Peruvian species of Vampyrops (Chiroptera: Phyllostomatidae). Jour. Mammal. 53 (1): 72-82.

Genoways, H.H. \& S.L. Williams. 1984. Results of the Alcoa Foundation - Suriname Expeditions. IX. Bats of the genus Tonatia (Mammalia: Chiroptera) in Suriname. Ann. Carnegie Mus. Nat. Hist. 53: $327-346$.

Genoways, H.H. \& S.L. Williams. 1986. Results of the Alcoa Foundation - Suriname Expeditions. XI. Bats of the genus Micronycteris (Mammalia: Chiroptera) in Suriname. Ann. Carnegie Mus. Nat. Hist. 55: 303-324.

Goodwin, G.G. 1942. A summary of recognizable species of Tonatia, with descriptions of two new species. Jour. Mammal. 23: $204-209$.

- 1963. American bats of genus Vampyressa, with description of a new species. Amer. Mus. Novit. 2125: 1-24.

Goodwin, G.G. \& A.M. GreEnhall. 1961. A review of the bats of Trinidad and Tobago. Bull. Amer. Mus. Nat. Hist. 122 (3): 187-302.

Greenhall, A.M.; G. Joermann; U. Schmidt \& M.R. Seidel. 1983. Desmodus rotundus. Mamm. Species 202: 1-6.

Greenhall, A.M.; U. Schmidt \& G. Joermann. 1984. Diphylla ecaudata. Mamm. Species 227: 1-3.

GREgorin, R. 1998. Extending geographic distribution of Chiroderma doriae Thomas 1891

(Phyllostomidae, Stenodermatinae). Chiroptera Neotropical 4 (2): 98-99.

Handley JR., C.O. 1976. Mammals of the Smithsonian Venezuelan Project. Brigham Young Univ. Sci. Bull., Biol. Ser., 20 (5): 1-91.

-1984. New species of mammals from northern South America: a long-tongued bat, genus Anoura Gray. Proc. Biol. Soc. Wash. 97 (3): 513-521.

- 1989. The Artibeus of Gray, 1838, p. 443-468. In: K.H. REDFORD \& J.F. EISENBERG (Eds). Advances in Neotropical Mammalogy. Gainesville, Sandhill Crane Press, 614p.

Husson, A.M. 1962. The bats of Suriname. Zool. Verh. Rijksmus. Nat. Hist., Leiden, 58: 1-282.

IвAм. 1998. Guia das Unidades de Conservação Ambiental do Rio de Janeiro. Rio de Janeiro, IBAM/DUMA/PCRJ/SMAC, 208p.

JONES JR., J.K., \& D.C. CARTER. 1976. Annotated checklist, with keys to subfamilies and genera. Pp. 7-38. In: R.J. BAKer; J.K. Jones JR. \& D.C. CARTER (Eds). Biology of bats of the New World family Phyllostomatidae, Part I. Spec. Publ. Mus., Texas Tech Univ. 10: 1-218.

Koepcke, J. \& R. Kraft. 1984. Cranial and external characters of the larger fruit bats of the genus Artibeus from Amazonian Peru (Chiroptera: Phyllostomidae). Spixiana 7 (1): 75-84.

Koopman, K.F. 1978. Zoogeography of Peruvian bats with special emphasis on the role of the Andes. Am. Mus. Novit. 2651: 1-33.

. 1982. Biogeography of the bats of South America, p. 273-302. In: M.A. MARES \& H.H. Genoways (Eds). Mammalian biology in South America. Spec. Publ. Ser., Pymatuning Lab. Ecol. 6: XII+1-539.

- 1993. Order Chiroptera, p.137-241. In: D.E. WILSON \& D.M. REEDER (Eds). Mammal species of the world: a taxonomic and geografic reference. Washington, D.C., Smithsonian Inst. Press, $2^{\text {nd }}$ ed., XVIII +539 p.

1994. Chiroptera: systematics. Handbuch der Zoologie [Handbook of Zoology], VIII (Mammalia) 8 (60): I+VII+217p.

Laval, R.K. 1973. A revision of the neotropical bats of the genus Myotis. Nat. Hist. Mus. L. Angeles Count. Sci. Bull. 15: 1-54.

LEWIS, S.E. \& D.E. WILSON. 1987 Vampyressa pusilla. Mamm. Species 292: 1-5. 
LiM, B.K. \& D.E. WiLson. 1993. Taxonomic status of Artibeus amplus (Chiroptera: Phyllostomidae) in northern South America. Jour. Mammal. 74 (3): 763-768.

Lima, J.L. 1926. Os morcegos do Museu Paulista. Rev. Mus. Paulista, São Paulo, 14: 42-127.

MARES, M.A.; M.R. WILLIG; K.E. STREILEIN \& J.R. LACHER. 1981. The mammals of northeastern Brazil: a preliminary assessment. Ann. Carnegie Mus. 50: 81-137.

Mares, M.A.; R.M. Barquez \& J.K. Braun. 1995. Distribution and ecology of some Argentine bats (Mammalia). Ann. Carnegie Mus. Nat. Hist. 64 (3): 219-237.

Mares, M.A.; R.M. Barquez; J.K. Braun \& R.A. Ojeda. 1996. Observations on the mammals of Tucumán Province, Argentina. I. Systematics, distribution, and ecology of the Didelphimorphia, Xenarthra, Chiroptera, Primates, Carnivora, Perissodactyla, Artiodactyla, and Lagomorpha. Ann. Carnegie Mus. Nat. Hist. 65 (2): 89-152.

MARINHO-FILHO, J. 1996. Distribution of bat diversity in the southern and southeastern Brazilian Atlantic Forest. Chiropt. Neotrop. 2 (2): 51-54.

MARques-Aguiar, S.A. 1994. A systematic review of the large species of Artibeus Leach, 1821 (Mammalia: Chiroptera) with some phylogenetic inferences. Bol. Mus. Paraense Emílio Goeldi, Ser. Zool., 10 (1): 1-83.

McLellan, L.J. 1984. A morphometric analysis of Carollia (Chiroptera, Phyllostomidae). Amer. Mus. Novit. 2791: 1-35.

Medellín, R.A. 1989. Chrotopterus auritus. Mamm. Species 343: 1-5.

Medellín, R.A. \& H. Arita. 1989. Tonatia evotis and Tonatia silvicola. Mamm. Species 334: 1-5.

Myers, P. \& R.M. Wetzel. 1979. New records of mammals from Paraguay. Jour. Mammal. 60 (3): 638-641.

1983. Systematics and zoogeography of bats of the Chaco Boreal. Misc. Publ. Mus. Zool. Univ. Michigan 165: 1-59.

NowAK, R.M. 1991. Walker's mammals of the world. Baltimore, Johns Hopkins Univ. Press, $5^{\text {th }}$ ed., vol. 1, I-XIV+642p.

Oliveira, R.F.; A.A. MaiA; T.M.P.A. Penna \& Z.M.S. Cunha. 1980. Estudo sobre a flora e a fauna da Represa do Camorim e áreas circunvizinhas. Rio de Janeiro, Fundação Estadual de Engenharia do Meio Ambiente/ DIPEC, relatório mimeografado, 43p.

Oliveira, R.R.; D.F. Lima; M.C. Vianna; I.M. Silva; P. Delamonica. 1995. Levantamento florístico do Parque Estadual da Pedra Branca. Relatório de acompanhamento. Rio de Janeiro, Fundação Estadual de Engenharia do Meio Ambiente, 29p.

Owen, R.D. 1988. Phenetic analyses of the bat subfamily Stenodermatinae (Chiroptera: Phyllostomidae). Jour. Mammal. 69 (4): 795-810.

Pacheco, V. \&. B.D. Patterson. 1991. Phylogenetic relationships of the New World bat genus Sturnira (Chiroptera: Phyllostomidae). Bull. Amer. Mus. Nat. Hist. 206: 101-121.

PEDRo, W.A. \& F.C. PAssos. 1995. Occurrence and food habits of some bat species from the Linhares Forest Reserve, Espirito Santo, Brazil. Bat Research News 36: 1-2.

Peracchi, A.L. \& S.T. Albuquerque. 1971. Lista provisória dos quirópteros dos Estados do Rio de Janeiro e Guanabara, Brasil (Mammalia, Chiroptera). Rev. Brasil. Biol. 31 (3): 405-413.

— 1986. Quirópteros do Estado do Rio de Janeiro, Brasil (Mammalia, Chiroptera). Anais do VII Congresso Brasileiro de Zoologia. Publ. Avulsas Mus. Nac. Rio de Janeiro 66: 63-69.

-1993. Quirópteros do município de Linhares, Estado do Espírito Santo, Brasil (Mammalia, Chiroptera). Rev. Brasil. Biol. 53: 575-581.

Peterson, R.L. 1968. A new bat of the genus Vampyressa from Guyana, South America, with a brief systematic review of the genus. Life Sci. Contr., Royal Ontario Mus.73: 1-17.

Pine, R.H. 1972. The bats of the genus Carollia. Tech. Monog., Texas Agric. Exp. Station, Texas A \& M Univ. 8: 1-125.

Pine, R.H. \& A. Ruschi. 1976. Concerning certain bats described and recorded from Espirito Santo, Brasil. An. Inst. Biol. Univ. Nac. Autón. Mexico, ser. Zool., 47 (2): 183-196.

Reis, N.R.; A.L. Peracchi; M.F. Muller; E.A. Bastos \& E.S. Soares. 1996. Quirópteros do Parque 
Estadual do Morro do Diabo, São Paulo, Brasil (Mammalia-Chiroptera). Rev. Brasil. Biol. 56 (1): $87-92$

Rouk, C.S. \& D.C. CARTER. 1972. A new species of Vampyrops (Chiroptera: Phyllostomatidae) from South America. Occas. Pap. Mus. Texas Tech. Univ. 1: 1-7.

Ruschi, A. 1953. Morcegos do Estado do Espírito Santo. XVII. Família Phyllostomidae. Descrição das espécies: Lonchophylla mordax e Hemiderma perspicillatum, com algumas observações biológicas a respeito. Bol. Mus. Biol. Prof. Mello Leitão 19: 1-7.

Sanborn, C.C. 1933. Bats of the genera Anoura and Lonchoglossa. Field Mus. Nat. Hist. Publ., Zool. ser., 20: 23-27.

- 1941. Descriptions and records of Neotropical bats. Field Mus. Nat. Hist. Publ., Zool. ser., 27: 371-387.

- 1949. Bats of the genus Micronycteris and its subgenera. Fieldiana Zool. 31: 215-233. 1955. Remarks on the bats of the genus Vampyrops. Fieldiana Zool. 37: 403-413.

SAzima, I.; L.D. Vizotto \& V.A. TAdDEI. 1978. Uma nova espécie de Lonchophylla da Serra do Cipó, Minas Gerais, Brasil (Mammalia, Chiroptera, Phyllostomidae). Rev. Brasil. Biol. 38 (1): 81-89.

SilveirA, E.K.P. 1965. Ocorrência de mamíferos da fauna original nas áreas do Sudeste brasileiro. Bol. Geogr., Rio de Janeiro, 187; 626-641.

Simmons, N.B. 1996. A new species of Micronycteris (Chiroptera: Phyllostomidae) from Northeastern Brazil, with comments on phylogenetic relationships. Amer. Mus. Novit. 3158: 1-34.

Simmons, N.B. \& R.S. Voss. 1998. The mammals of Paracou, French Guiana: a neotropical lowland rainforest fauna. Part I. Bats. Bull. Amer. Mus. Nat. Hist. 273: 1-219.

Swanepoel, P. \& H.H. Genoways. 1979. Morphometrics, p. 13-106. In: R.J. Baker; J.K. Jones JR. \& D.C. CArter (Eds). Biology of bats of the New World family Phyllostomatidae, Part III. Spec. Publ. Mus., Texas Tech Univ. 16: 1-441.

TAddel, V.A. 1975a. Phyllostomatidae (Chiroptera) do Norte-Ocidental do Estado de São Paulo. I Phyllostominae. Ciênc. Cult. 27 (6): 621-632.

_ 1975b. Phyllostomatidae (Chiroptera) do Norte-Ocidental do Estado de São Paulo. II Glossophaginae; Carollinae; Sturnirinae. Ciênc. Cult. 27 (7): $723-734$.

— 1979. Phyllostomatidae (Chiroptera) do Norte-Ocidental do Estado de São Paulo. III -Stenodermatinae. Ciênc. Cult. 31 (8): 900-914.

TAddei, V.A.; L.D. VizotTo \& I. Sazima. 1983. Uma nova espécie de Lonchophylla do Brasil e chave para identificação das espécies do gênero (Chiroptera, Phyllostomidae). Ciênc. Cult. 35 (5): 625-629.

TAdDEI, V.A.; S.A. SouZA \& J.L. MAnUZzI. 1988. Notas sobre uma coleção de Lonchophylla bokermanni de Ilha Grande, Sudeste do Brasil (Mammalia, Chiroptera). Rev. Brasil. Biol. 48 (4): 851-855.

TAmsitT, J.R. \& D. Valdivieso. 1966. Taxonomic comments of Anoura caudifer, Artibeus lituratus and Molossus molossus. Jour. Mammal. 47 (2): 230-238.

Teixeira, S.C. \& A.L. Peracchi. 1996. Morcegos do Parque Estadual da Serra da Tiririca, Rio de Janeiro, Brasil (Mammalia, Chiroptera). Revta bras. Zool. 13 (1): 61-66.

Thomas, O. 1901. On a collection of bats from Para. Ann. Mag. Nat. Hist. (7) 8: 189-193.

VAZ, S.M. 1985. Mamíferos do Rio de Janeiro. Bol. Fund. Brasil. Cons. Nat., Rio de Janeiro, 20: 80-89

VIEIRA, C.O.C. 1942. Ensaio monográfico sobre os quirópteros do Brasil. Arquivos Zool. Estado São Paulo 3 (8): 219-471.

- 1955. Lista remissiva dos mamíferos do Brasil. Arquivos Zool. Estado São Paulo 8 : 341-474.

VizotTo, L.D. \& V.A. TADDEI. 1973. Chave para determinação de quirópteros brasileiros. Revta. Fac. Filos. Ciênc. Letras Bol. Ciênc., São José do Rio Preto, 1: 1-72.

Webster, W.D. 1993. Systematics and evolution of bats of the genus Glossophaga. Spec. Publ. Mus., Texas Tech Univ. 36: 1-184.

WiLliams, D.F. 1978. Taxonomic and karyologic comments on small brown bats, genus Eptesicus, from South America. Ann. Carnegie Mus. Nat. Hist. 47 (16): 361-383. 
Williams, S.L. \& H.H. GenOways. 1980. Results of the Alcoa Foundation - Suriname Expeditions. II. Additional records of bats (Mammalia: Chiroptera) from Suriname. Ann. Carnegie Mus. Nat. Hist. 49 (15): 213-236.

Williams, S.L., M.R. Willig \& F.A. REID. 1995. Review of the Tonatia bidens complex (Mammalia: Chiroptera), with descriptions of two new subspecies. Jour. Mammal. 76 (2): 612-626.

WiLLIG, M.R. 1983. Composition, microgeographic variation, and sexual dimorphism in Caatingas and Cerrado bat communities from northeast Brazil. Bull. Carnegie Mus. Nat. Hist. 23: 1-131.

WiLLIG, M.R \& M.A. MARES. 1989. Mammals from the caatinga: an updated list and summary of recent research. Rev. Brasil. Biol. 49 (2): 361-367.

Willig, M.R. \& R.R. Hollander. 1987. Vampyrops lineatus. Mamm. Species 275: 1-4.

WILSON, D.E. 1996. Neotropical bats: a checklist with Conservation Status, p: 167-177. In: A.C. GIBSON

(Ed.). Neotropical Biodiversity and Conservation. Occas. Publ. Mildred E. Mathias Botanical Garden, Los Angeles, 1: 1-202.

Recebido em 29.VII.2002; aceito em 18.XI.2002. 